Research Paper

\title{
The MEK inhibitor U0126 ameliorates diabetic cardiomyopathy by restricting XBPI's phosphorylation dependent SUMOylation
}

Tao Wang1,2\#, Jinhua Wu1,3\#, Wei Dong4,5,6,7\#, Mengwen Wang1, Xiaodan Zhong1, Wenjun Zhang1, Lei Dai ${ }^{1}$, Yang Xie', Yujian Liu1, Xingwei Heㄹ, Wanjun Liu ${ }^{1}$, Thati Madhusudhan ${ }^{8}$, Hesong Zeng ${ }^{1}{ }^{凶}$ and Hongjie Wang ${ }^{1 凶}$

1. Division of Cardiology, Department of Internal Medicine, Tongji Hospital, Tongji Medical College, Huazhong University of Science and Technology, Wuhan, 430030, PR China; Hubei Key Laboratory of Genetics and Molecular Mechanisms of Cardiological Disorders, Wuhan, 430030, PR China

2. Department of Cardiology, Affiliated Hospital of Weifang Medical University, Weifang, Shandong, 261000, PR China

3. Departments of Respiratory and Critical Care Medicine, Guangdong Provincial People's Hospital, Guangzhou, 510000, PR China

4. Hepatic Surgery Center, Tongji Hospital, Tongji Medical College, Huazhong University of Science and Technology, Wuhan, 430030, PR China

5. Hubei Key Laboratory of Hepato-Pancreato-Biliary Diseases, Wuhan, Hubei, 430030, PR China

6. Hubei Clinical Medicine Research Center of Hepatic Surgery, Wuhan, Hubei, 430030, PR China

7. Key Laboratory of Organ Transplantation, Ministry of Education; NHC Key Laboratory of Organ Transplantation; Key Laboratory of Organ

Transplantation, Chinese Academy of Medical Sciences, Wuhan, Hubei, 430030, PR China

8. Center for Thrombosis and Hemostasis, University Medical Center Mainz, Langenbeckstr. 1, 55131 Mainz, Germany

\#T.W., J.W. and W.D. contribute equally to this manuscript.

$\triangle$ Corresponding authors: Hesong Zeng, MD, PHD, Division of Cardiology, Department of Internal Medicine, Tongji Hospital, Tongii Medical College, Huazhong University of Science and Technology No.1095 Jiefang Road, Wuhan, Hubei 430030, PR China. Tel: +86-27-8369-2479; Fax: +86-27-8366-2843; Email: zenghs@tjh.tjmu.edu.cn. Hongjie Wang, MD, PHD, Division of Cardiology, Department of Internal Medicine, Tongji Hospital, Tongji Medical College, Huazhong University of Science and Technology No.1095 Jiefang Road, Wuhan, Hubei 430030, PR China. Tel: +86-27-8369-2479; Fax: +86-27-8366-2843; Email: hongjie.wang@tjh.tjmu.edu.cn

(C) The author(s). This is an open access article distributed under the terms of the Creative Commons Attribution License (https://creativecommons.org/licenses/by/4.0/). See http://ivyspring.com/terms for full terms and conditions.

Received: 2021.03.15; Accepted: 2021.06.19; Published: 2021.07.13

\begin{abstract}
Background: Chronic diabetes accelerates vascular dysfunction often resulting in cardiomyopathy but underlying mechanisms remain unclear. Recent studies have shown that the deregulated unfolded protein response (UPR) dependent on highly conserved IREla-spliced X-box- binding protein (XBPIs) and the resulting endoplasmic reticulum stress (ER-Stress) plays a crucial role in the occurrence and development of diabetic cardiomyopathy (DCM). In the present study, we determined whether targeting MAPK/ERK pathway using MEK inhibitor U0126 could ameliorate DCM by regulating IREl $\alpha-X B P I s$ pathway.

Method: Three groups of 8-week-old C57/BL6] mice were studied: one group received saline injection as control $(n=8)$ and two groups were made diabetic by streptozotocin (STZ) $(n=10$ each). 18 weeks after STZ injection and stable hyperglycemia, one group had saline treatment while the second group was treated with U0126 $(1 \mathrm{mg} / \mathrm{kg} /$ day), 8 weeks later, all groups were sacrificed. Cardiac function/histopathological changes were determined by echocardiogram examination, Millar catheter system, hematoxylin-eosin staining and western blot analysis. $\mathrm{H} 9 \mathrm{C} 2$ cardiomyocytes were employed for in vitro studies.

Results: Echocardiographic, hemodynamic and histological data showed overt myocardial hypertrophy and worsened cardiac function in diabetic mice. Chronic diabetic milieu enhanced SUMOylation and impaired nuclear translocation of XBPIs. Intriguingly, U0126 treatment significantly ameliorated progression of DCM, and this protective effect was achieved through enriching XBP1s' nuclear accumulation. Mechanistically, U0126 inhibited XBP1s' phosphorylation on S348 and SUMOylation on K276 promoting XBP1s' nuclear translocation. Collectively, these results identify that MEK inhibition restores XBPIs-dependent UPR and protects against diabetes-induced cardiac remodeling.
\end{abstract}

Conclusion: The current study identifies previously unknown function of MEK/ERK pathway in regulation of ER-stress in DCM. U0126 could be a therapeutic target for the treatment of DCM.

Key words: XBP1s; diabetic cardiomyopathy; endoplasmic reticulum stress; SUMOylation; U0126 


\section{Background}

Diabetes mellitus (DM) is one of the major chronic diseases endangering people's health worldwide. It has two major subtypes, type 1 (T1DM) and type 2 (T2DM). Cardiovascular disease (CVD) is a major cause of morbidity and mortality in diabetic patients. Patients with diabetes mellitus have a risk of cardiovascular events that is two to three times as high as the risk among those without diabetes[1, 2]. Interestingly, a subset of diabetic patients develops left ventricular dysfunction in the absence of coronary artery disease, hypertension or vascular disease. This observation, first made by Rubler et al. in 1972, is now known as diabetic cardiomyopathy (DCM)[3]. DCM is defined by the existence of abnormal myocardial structure and performance in the absence of other cardiac risk factors, such as coronary artery disease, hypertension, and significant valvular disease, in individuals with diabetes mellitus[4]. Their histopathological studies revealed diffuse fibrosis, myofibrillar hypertrophy, microvascular disease and deposition of acid mucopolysaccharide material[3, 4].

With improvements in clinical treatments, the relative risk of death declined by $29 \%$ over a 10 -year period, however, mortality in T1DM still increased 2to 8- fold. Early onset T1DM is a strong risk factor associated with serious CVD, moreover, with a significant higher risk level in women, they also die around 18 years earlier than their diabetes free counterparts[5-8]. In compared with T2DM, several studies revealed that T1DM patients have an even higher incidence of heart failure (HF), when disease duration is $>20$ years, CVD becomes the primary cause of death[9, 10]. In addition, because of the incongruent clinical features the underlying mechanisms of CVD may be partially distinct in T1DM versus T2DM[11]. However, CVD in T2DM and T1DM is often considered the same disease, with the diagnosis and treatment of CVD in T1DM extrapolated from the experience with T2DM. Thus, it has become challenging to identify T1DM-specific mechanisms to develop T1DM-specific therapies for DCM[12-14].

As reported in the literature, increases in oxidative stress, reduced nitric oxide bioavailability, elevations in advanced glycation end products, mitochondrial dysfunction and collagen-based cardiomyocyte and extracellular matrix stiffness, inflammation, renin-angiotensin-aldosterone system activation, cardiac autonomic neuropathy, endoplasmic reticulum (ER) stress, microvascular dysfunction and a myriad of cardiac metabolic abnormalities have all been implicated in the development and progression of DCM[4, 15]. Among these signaling pathways, ER stress pathway is continuously the studying focus and the three principle pathways of the unfolded protein response (UPR), including IRE1-XBP1, PERK-ATF4 and ATF6, are widely studied $[16,17]$. XBP1, a key transcription factor of the UPR, is critical in maintaining ER homoeostasis and is activated by disturbances in endoplasmic reticulum (ER) protein-folding homeostasis. In response to upstream signals, XBP1 mRNA undergoes an unconventional splicing by the ER transmembrane endoribonuclease IRE1, which ultimately generates the potent transcriptional transactivator $\mathrm{XBP} 1 \mathrm{~s}$. $\mathrm{XBP} 1 \mathrm{~s}$ promotes ER biogenesis and activates the expression of ER chaperone genes that are required for the folding and trafficking of secretory cargo proteins[18]. It plays a pivotal role in cardiac pathological regulation, such as cardiac ischemic reperfusion injury, transverse aortic constriction induced hypertrophy, etc. However, the $\mathrm{XBP1s}$ role in DCM remains largely unknown[19]. Moreover, our previous study showed that the XBP1 signaling pathway is required for an adaptive ER-response in diabetic nephropathy (DN), while genetic disruption or functional inactivation of this pathway in diabetic mouse models promotes a maladaptive UPR. The latter is hallmarked by impaired spliced XBP1 (XBP1s) nuclear translocation, which provokes a maladaptive ER-response characterized by ATF6 and CHOP signaling in $\mathrm{DN}[20]$. To this end, we proposed a vital role of XBP1s in cardiomyocytes in the context of maladaptive ER-response under DCM condition.

Furthermore, post-translational modification of XBP1, especially its SUMOylation has been shown to be involved in UPR regulation. Chen H. et al. reported that XBP1 can be SUMOylated by PIAS2 (protein inhibitor of activated STAT2) at two lysine residues located in the C-terminal transactivation domain. These SUMOylation events significantly decrease the transcriptional activity of XBP1 towards UPR target genes[21]. While others showed that Sentrin/SUMO-specific protease 1 (SENP1), a specific de-SUMOylation protease for XBP1 can increase the transcriptional activity of $\mathrm{XBP} 1$ and ER stress-induced apoptosis through accumulating SUMOylated XBP1 $[22,23]$. Thus, we speculated that SUMOylation of XBP1 may regulate UPR activation and ER homeostasis in DCM.

Within the current study, we confirmed a correlation of $\mathrm{XBP} 1 \mathrm{~s}^{\prime}$ nuclear translocation in cardiomyocyte and DCM phenotype in a streptozotocin induced type 1 mouse DM model. Furthermore, we found that the impaired XBP1s' nuclear translocation is regulated by its SUMOylation in the diabetic hearts. Subsequently, the molecular 
mechanisms underlying the regulation of the XBP1s' SUMOylation in cardiomyocyte were systematically investigated, and we found that high glucose induced activation of MAP kinase ERK1/2 can increase XBP1s' phosphorylation on its serine residue S348, which in turn can promote its SUMOylation on lysine residue K276 and result in its impaired nuclear translocation. Finally, we tested whether the ERK1/2 inhibitor U0126 treatment can protect against DCM, as hypothesized U0126 treatment could be of therapeutic potential for DCM.

\section{Materials and methods}

\section{Induction of diabetes using streptozotocin}

Animal experiments were conducted following standards and procedures approved by the local Animal Care and Use Committee (Tongji Medical College Experimental Animal Center, Huazhong University of Science and Technology, China). Diabetes was induced by intraperitoneal administration of streptozotocin (STZ, Sigma-Aldrich, St. Louis, MO) at $60 \mathrm{mg} / \mathrm{kg}$, freshly dissolved in 0.05 $\mathrm{M}$ sterile sodium citrate $(\mathrm{pH} 4.5)$ on five successive days in 8-week-old male C57BL/6J mice (HFK Bioscience, Beijing, China)[20]. Mice were considered diabetic if blood glucose levels determined from the tail vein using ONETOUCH glucose strips were above $300 \mathrm{mg} / \mathrm{dl}(16.7 \mathrm{mmol} / \mathrm{L})$ after the last STZ injection. Mice displaying blood glucose levels above $500 \mathrm{mg} / \mathrm{dl}(27.7 \mathrm{mmol} / \mathrm{L})$ received insulin Lantus (1$2 \mathrm{U}$, Sanofi, Beijing, China) to avoid excessive and potentially lethal hyperglycemia. We injected a subset of diabetic mice intraperitoneally with either U0126 (LC Laboratories, Woburn, MA) (1mg/ $\mathrm{kg}$, dissolved in $6 \%$ DMSO-PBS) or $6 \%$ DMSO-PBS once daily starting 18 weeks after the last STZ injection until 1 day before analysis [20]. Blood and tissue samples were obtained at 26 weeks after injection of STZ.

\section{Echocardiographic analysis}

Mice were anesthetized using light isoflurane (1$2 \%)$. Echocardiography was performed as described previously using a high-resolution imaging system with a 13-MHz linear ultrasound transducer (VisualSonics Vevo770, VisualSonics Inc., Toronto, Canada).

\section{Hemodynamic measurements of left ventricular (LV) function}

Measurements of LV function were performed using Millar catheter system as described before. In brief, mice were anaesthetized, and a pressurevolume catheter (Millar 1.4F, SPR 835, Millar Instruments, Inc. Houston, TX, USA) was inserted into the right carotid artery and advanced into the left ventricle under pressure control to measure instantaneous intraventricular pressure and volume.

\section{Histology and immunohistochemistry}

Histology analysis was performed as previously described[20, 24]. We first perfused freshly sacrificed mice with ice-cold PBS and then with $4 \%$ paraformaldehyde. Tissues were further fixed in $4 \%$ paraformaldehyde for 48 hours, embedded in paraffin and processed for sectioning. Cardiomyocyte area was quantified in sections stained with H\&E (JianCheng, Nanjing, China) and wheat germ agglutinin (WGA) (Vector Laboratories, Burlingame, CA). Images were also captured with a Nikon-Microscope (NIKON, Tokyo, Japan). The outline of myocytes was traced using Image J software to determine myocyte cross-sectional area in the LV of each animal. A value from each heart was calculated by measuring about $400-600$ cells in a remote area from 5 randomly selected image areas in an individual heart.

\section{Cell culture and transfection}

H9C2 cells (ATCC) were cultured in RPMI 1640 medium (Sigma-Aldrich, St. Louis, MO) supplemented with $10 \%$ Fetal bovine serum (FBS, Life Technologies, UK) and Penicillin/Streptomycin (P/S) $(100 \mathrm{IU} / \mathrm{mL})$ in a humidified atmosphere of $95 \%$ air and $5 \% \mathrm{CO} 2$ at $37^{\circ} \mathrm{C}$. HEK293T cells (ATCC) were maintained in DMEM medium supplemented with $10 \%$ FBS. Cells were starved overnight before treatment with high concentrations of glucose (HG, 25 $\mathrm{mM})$ or mannitol $(25 \mathrm{mM})$. Transfection was performed using transfection reagent FuGENE (Promega, Germany) according to the manufacturer's protocol. At desired time points post glucose and mannitol treatment, total lysates or cytosolic and nuclear fractions of cells were prepared for immunoblotting analysis.

\section{Cell fractionation}

Isolation of cytosolic and nuclear fractions was performed as previously described[20]. Heart samples were lysed using tissue homogenizer in buffer-A containing $10 \mathrm{mM}$ HEPES-KOH (pH 7.9), $10 \mathrm{mM} \mathrm{KCl,}$ $1.5 \mathrm{mM} \mathrm{MgCl} 2,1 \mathrm{mM}$ EDTA, $0.6 \% \mathrm{NP}-40,0.5 \mathrm{mM}$ DTT, protease inhibitor cocktail (Roche diagnostics $\mathrm{GmbH}$, Mannheim, Germany) and lysates were incubated for $10 \mathrm{~min}$ on ice. After brief vortexing the lysates were centrifuged for $10 \mathrm{~min}$ at 5,000 rpm at $4^{\circ} \mathrm{C}$. Supernatants were collected as cytosolic fractions and the pellets were resuspended in $100 \mathrm{ml}$ of buffer-B containing $10 \mathrm{mM}$ HEPES-KOH ( $\mathrm{pH}$ 7.9), $25 \%$ glycerol, $420 \mathrm{mM} \mathrm{NaCl}, 1.5 \mathrm{mM} \mathrm{MgCl} 2,0.2 \mathrm{mM}$ EDTA, $0.5 \mathrm{mM}$ DTT and protease inhibitors. Lysates were incubated for $20 \mathrm{~min}$ on ice followed by 
centrifugation at $12,000 \mathrm{~g}$ at $4^{\circ} \mathrm{C}$ for $10 \mathrm{~min}$. Supernatants containing the nuclear extracts were collected and stored at $-80^{\circ} \mathrm{C}$.

A similar procedure was used for isolation of cytosolic and nuclear fractions of cells where buffer-A contains $10 \mathrm{mM}$ HEPES-KOH ( $\mathrm{pH} 7.9), 10 \mathrm{mM} \mathrm{KCl}$, $1.5 \mathrm{mM} \mathrm{MgCl} 2,0.5 \mathrm{mM}$ DTT and protease inhibitors. Protein concentration was measured using Bradford reagent or BCA reagent (Boster, Wuhan, China), and purity of nuclear and cytoplasmic fractions was determined by Lamin A/C (Santa Cruz Biotechnology, Inc) and GAPDH (Cell Signaling Technology, Danvers, MA) western blots, respectively.

\section{Immunoblotting and Immunoprecipitation}

Immunoblotting and Immunoprecipitation were performed as previously described[20, 25]. In brief, for immunoblotting total lysates were prepared in RIPA buffer (50 mM Tris at pH 7.4, 1\% NP-40, 0.25\% sodium deoxycholate, $150 \mathrm{mM} \mathrm{NaCl}, 1 \mathrm{mM}$ EDTA, and $1 \mathrm{mM} \mathrm{Na} \mathrm{VO}_{4}$, supplemented with protease inhibitor cocktail). Lysates were centrifuged $(12,000 \times$ $\mathrm{g}$ for $20 \mathrm{~min}$ at $4{ }^{\circ} \mathrm{C}$ ) and supernatant was kept, while the pellet containing debris was discarded. The protein concentration in supernatants was quantified using BCA reagent. Equal amounts of protein were electrophoretically separated on $10 \%$ or $12.5 \%$ SDS polyacrylamide gels, transferred to PVDF membranes, and probed with the desired primary antibodies overnight at $4{ }^{\circ} \mathrm{C}$. Membranes were then washed with TBST and incubated with anti-mouse or anti-rabbit IgG (1:2000, Abcam, Cambridge, MA) horseradish peroxidase-conjugated antibodies as indicated. Blots were developed with the immobilon western chemiluminiscent HRP substrate. The density of each band was measured by using Image software.

For Immunoprecipitation of total cellular and heart tissue proteins, proteins were extracted with RIPA containing complete protease inhibitor cocktail. Lysates were combined with $1 \mu \mathrm{g}$ of specific antibody and incubated overnight at $4{ }^{\circ} \mathrm{C}$ on rotating shaker. Immunoprecipitates were purified with protein $A / G$ agarose beads (Santa Cruz, CA) and washed with PBS containing protease inhibitor cocktail. Immunoprecipitates were fractionated by SDS-PAGE (10\%), transferred to PVDF membranes, and subjected to immunoblotting with appropriate primary and secondary antibodies as described above. The following antibodies were used in the current study: ANP (Thermo Fisher Scientific, Rockford, USA), Flag, XBP-1, BNP (Santa Cruz Biotechnology, Inc), SUMO-1, SUMO2/3, ERK1/2, P-ERK1/2, P38, p-P38, JNK, p-JNK (Cell Signaling Technology, Danvers, MA), $\beta$-MHC (Abcam, Cambridge, MA).

\section{Plasmid DNA, Adenovirus and Quick-change mutagenesis}

Plasmids encoding wildtype or lysine mutant XBP1s-GFP fusion (K276R, K297R and K298R XBP1s) proteins were gifts from Dr. Ling Qi (Cornell University)[21]. Mouse XBP1s mutations (Ser61Ala, Ser169Ala, and Ser348Ala) were achieved using the Quick Change Site-Directed Mutagenesis Kit (Stratagene, La Jolla, CA) with the following primers respectively: Ser61Ala: 3'-GCTCACGCACCTGGCC CCGGAGGAGAAAG-5' (F), 5'-CTTTCTCCTCCGGG GCCAGGTGCGTGAGC'-3(R); Ser169Ala: 3'-GCCC AGTTGTCACCGCCCCAGAACATCTTC-5' (F), 5'-G AAGATGTTCTGGGGCGGTGACAACTGGGC-3'(R); Ser348Ala: 3'-CTTCAGTGACATGTCTGCTCCACTT GGTACAG-5' (F), 5'-CTGTACCAAGTGGAGCAGA CATGTCACTGAAG-3' (R); All mutants were confirmed by sequencing. Ad-EGFP, Ad-XBP1s, Ad-K276R XBP1s, Ad-K297R XBP1s, Ad-K298R XBP1s and Ad-S348A XBP1s (Shanghai DesignGene Biotechnology Co., Ltd) were carried out in H9C2 cells for 24 hours. Adenoviruses were transduced at 25 multiplicity of infection (MOI).

\section{Real Time PCR}

To examine the downstream UPR genes in wild type and K276R mutant transfected H9C2 cells, we performed UPR pathway-specific expression analyses. Quantitative RT-PCR were conducted essentially as previously described[26]. Transfected H9C2 cells were thawed on ice and transferred into TRIZOL (Life Technologies, Shanghai, China) for isolation of total RNA following the manufacturer's protocol. Quality of total RNA was ensured on an agarose gel and by analyses of the A260/280 ratio. The reverse transcription reaction was conducted with $1 \mu \mathrm{g}$ of total RNA using the Super Script reagents and oligo (dT) primers (Sangon Biotech, Shanghai, China). cDNA was amplified using the primers listed in the Supplementary Table S1.

\section{Immunofluorescence}

Fluorescent microscopy was performed as previously described[21]. H9C2 cells split onto sterile cover glasses coated with poly-L-lysine (Sigma) were transfected with adenoviruses encoding wildtype or lysine mutant XBP1s-GFP fusion proteins and then incubated with HG for 6 hours. Cells cover glasses were removed from dishes and then counterstained with DAPI. The fluorescent images were taken with a Nikon Eclipse E600 microscope or a Zeiss Axiovert 135.

Immunofluorescence microscopy was performed as previously described[27]. Briefly, H9C2 cells on coverslips were fixed with $4 \%$ paraformaldehyde for 
15 min, then washed with PBS three times. The cells were blocked with normal goat serum and incubated with anti-XBP1s antibody overnight. Cells were washed with PBS three times and then incubated with secondary antibodies conjugated with Alexa Fluor 488 goat anti-mouse IgG antibody (Invitrogen) for 1 hour. Cells were washed with PBS three times and then counterstained with DAPI. Immunofluorescence images were taken with a Nikon Eclipse E600 microscope or a Zeiss Axiovert 135.

\section{Statistical analysis}

The data are summarized as the means \pm SEM (standard error of the mean). Statistical analyses were performed with Student's $t$-test or ANOVA as appropriate and post-hoc comparisons of ANOVA were corrected with the method of Tukey. Prism 5 (www.graphpad.com) software was used for statistical analyses. All data presented involving cell culture is representative of at least three independent repeat experiments. Statistical significance was accepted at values of $p<0.05$.

\section{Results}

\section{Diabetic mice displayed cardiac function impairment and left ventricular hypertrophy}

As described in materials and methods, we used a mouse model of persistent hyperglycemia induced by streptozotocin (STZ model), reflecting insulinopenic type $1 \mathrm{DM}$ (Figure 1A)[28]. After established diabetes, we detected random blood glucose in the indicated time points. Random blood glucose levels were significantly increased in STZ-induced mice compared with the control group (Figure 1B). As shown in Figure 1C, E, the myocardial structure was examined by Hematoxylin and eosin (H\&E) and WGA staining, diabetic hearts displayed structural abnormalities, including disrupted cardiac fibers, deranged cellular structures, obscured intercellular border and increased cardiomyocyte transverse cross-sectional areas (Figure 1D, F). Immunoblots showed an increase in the expression of atrial natriuretic peptide (ANP), B type natriuretic peptide (BNP) and $\beta$-myosin heavy chain $(\beta-\mathrm{MHC})$ in the heart tissues of diabetic cardiomyopathy mice (Figure 1G-H). To evaluate the changes of left ventricular performance in diabetic mice, M-mode echocardiography was performed at 26 weeks after STZ injection (Figure 1I). Compared to control, diabetic cardiomyopathy mice showed a significant reduction of ejection fraction and fraction shorting (Figure 1J-K). We also detected the left ventricular hemodynamic alternation simultaneously. Diabetic mice showed aggravated cardiac dysfunction, which was mainly manifested in the decline in $+\mathrm{dP} / \mathrm{dt} \max$ and $-\mathrm{dP} / \mathrm{dt}$ min (Figure 1L-M). These data indicated that the type 1 DCM was successfully established, characterized by diastolic and systolic dysfunction and myocardial hypertrophy.

\section{XBPIs' nuclear translocation was significantly reduced and negatively correlated with the level of its SUMOylation in diabetic mouse heart}

Chronic hyperglycemia in diabetes impairs nuclear translocation of XBP1s compromising UPR resulting in maladaptive ER-stress in diabetic kidney[20, 29]. In agreement with these observations, chronic diabetes induced impaired nuclear translocation of XBP1s in the diabetic heart. (Figure 2A, B). Besides, other brunches of the UPR were subsequently deregulated, such as ATF6 and CHOP (Supplementary Figure 1). Furthermore, in vitro studies with neonatal cardiomyocytes $\mathrm{H} 9 \mathrm{C} 2$ cells showed that high concentration glucose (HG, 25mM) but not mannitol impaired nuclear translocation of XBP1s (Figure 2C-D, Supplementary Figure 2). These data suggest that akin to diabetic kidney, nuclear translocation of XBP1s is impaired in diabetic heart promoting maladaptive ER-stress and cardiomyopathy.

SUMOylation of proteins, post-translational modification by the small ubiquitin-like modifier (SUMO), is an important transient regulatory mechanism in many cellular processes, most notably transcriptional regulation, DNA damage, and signal transduction[30, 31]. To identify whether SUMOylation of XBP1s modulates its protective functions in DCM, we conducted immunoprecipitation for total proteins extracted from cardiac tissue of control and DCM mice. We found that XBP1s protein was SUMOylated by SUMO1 and SUMO2/3 in DCM mice (Figure 2E, Supplementary Figure 3). Taken together, these data suggest that its SUMOylation could regulate the impaired XBP1s nuclear translocation observed in STZ-induced diabetic cardiomyopathy mice.

\section{HG inhibits XBPIs' nuclear translocation through SUMOylation of XBP Is at the lysine residue 276}

SUMOylation of transcription factors often leads to alterations in the intracellular localization of target proteins[30]. Therefore, we investigated the physiological significance of SUMOylation on XBP1s' nuclear translocation. Lysine residues K276 and K297 were identified as two highly-conserved SUMOylation motifs and located within the transactivation domain of the $\mathrm{XBP} 1 \mathrm{~s}$ protein, a region 
that is absent in the XBP1u (unspliced XBP1) protein[21]. This sequence is highly conserved across the different paralogous homologs in mouse, human, rat and other species (Supplementary Figure 4A). In order to exclude the possibility of non-specific modification events, mutation of K298 to R (K298R) which had no effect on SUMOylation of XBP1s protein that carried a negative charge. The scheme shown in Supplementary Figure 4A summarizes mutational loci of K276R, K297R and K298R. Next, we expressed XBP1s-GFP fusion proteins for wild type and lysine mutant XBP1s-GFP fusion (K276R, K297R and K298R) proteins in H9C2 cells (Supplementary Figure 4B-C). Interestingly, mutation of K276 to R (K276R) obviously increased XBP1s' nuclear translocation, while other mutations had no significant effect (Figure 3A, B). Consistent with this result, fluorescent microscopic images of XBP1s-GFP fusion adenovirus constructs upon 24 hours transfection into $\mathrm{H} 9 \mathrm{C} 2$ cells following $\mathrm{HG}$ intervention for 6 hours showed that mutant K276R predominantly localized to the nucleus (Figure 3C). To further prove its functional relevance in ER-stress, we examined the downstream UPR genes in wild type and K276R mutant transfected H9C2 cells simultaneously. In congruent with increased nuclear translocation of XBP1s, when compared to wild type, K276R mutant enhanced the expression of genes that modulate UPR (Figure 3D-E). Thus, our data suggest that $\mathrm{HG}$ inhibited $\mathrm{XBP} 1 \mathrm{~s}^{\prime}$ nuclear translocation through enhancing its SUMOylation at lysine residue 276 .
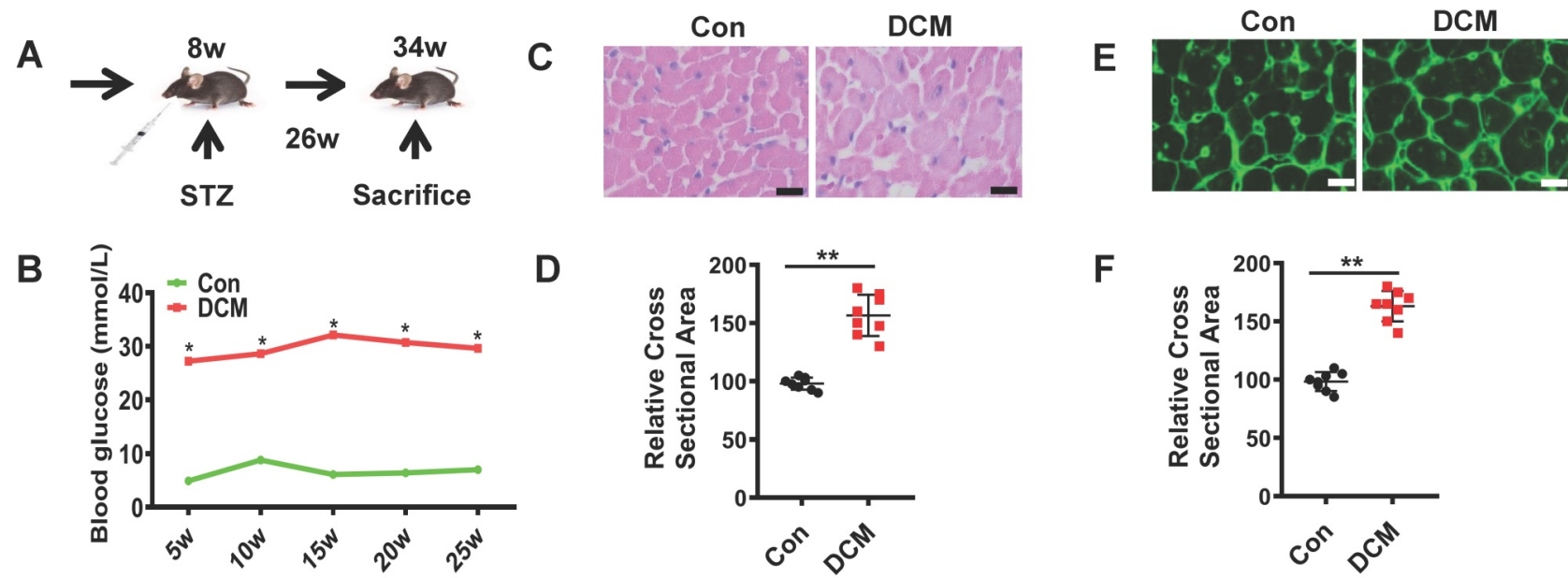

D

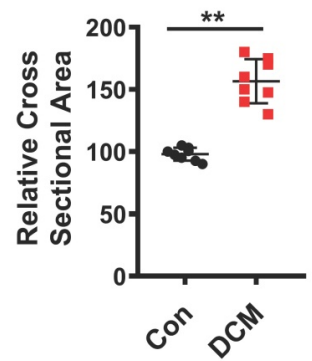

$\mathbf{F}$
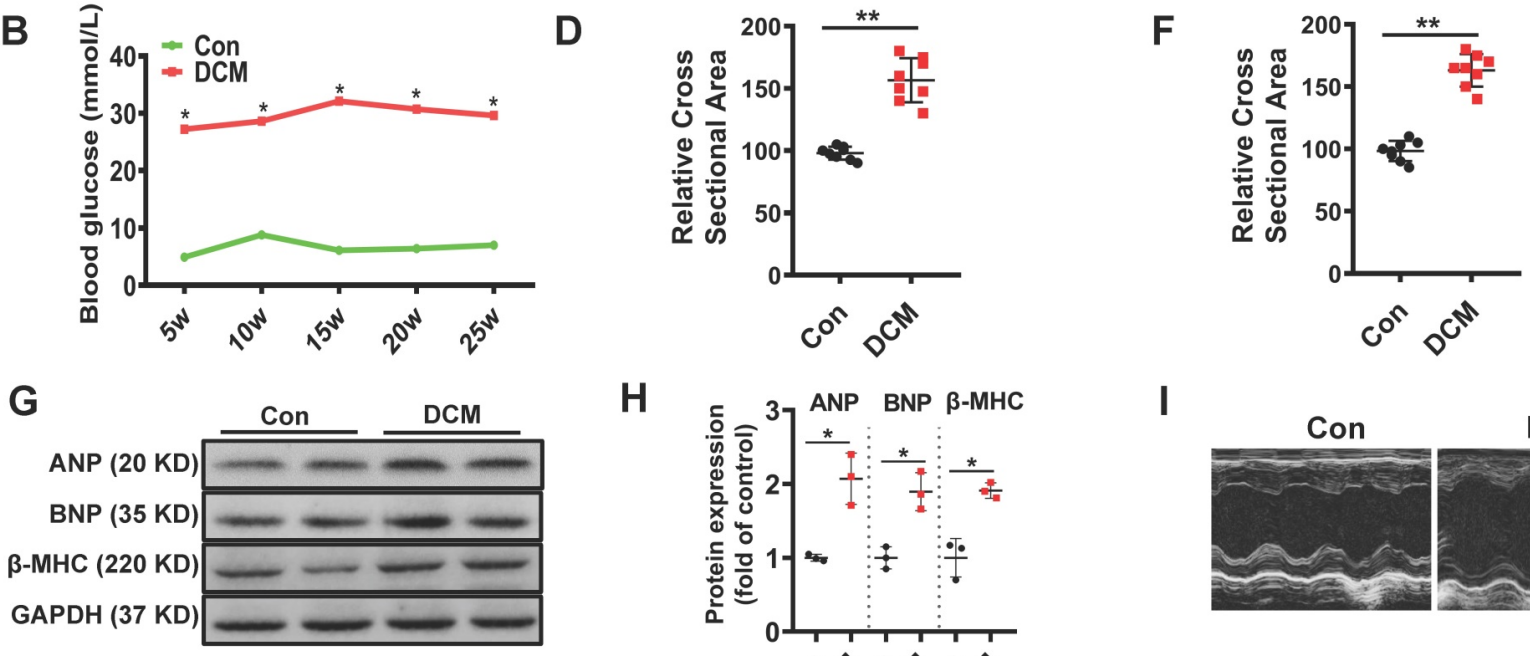

H
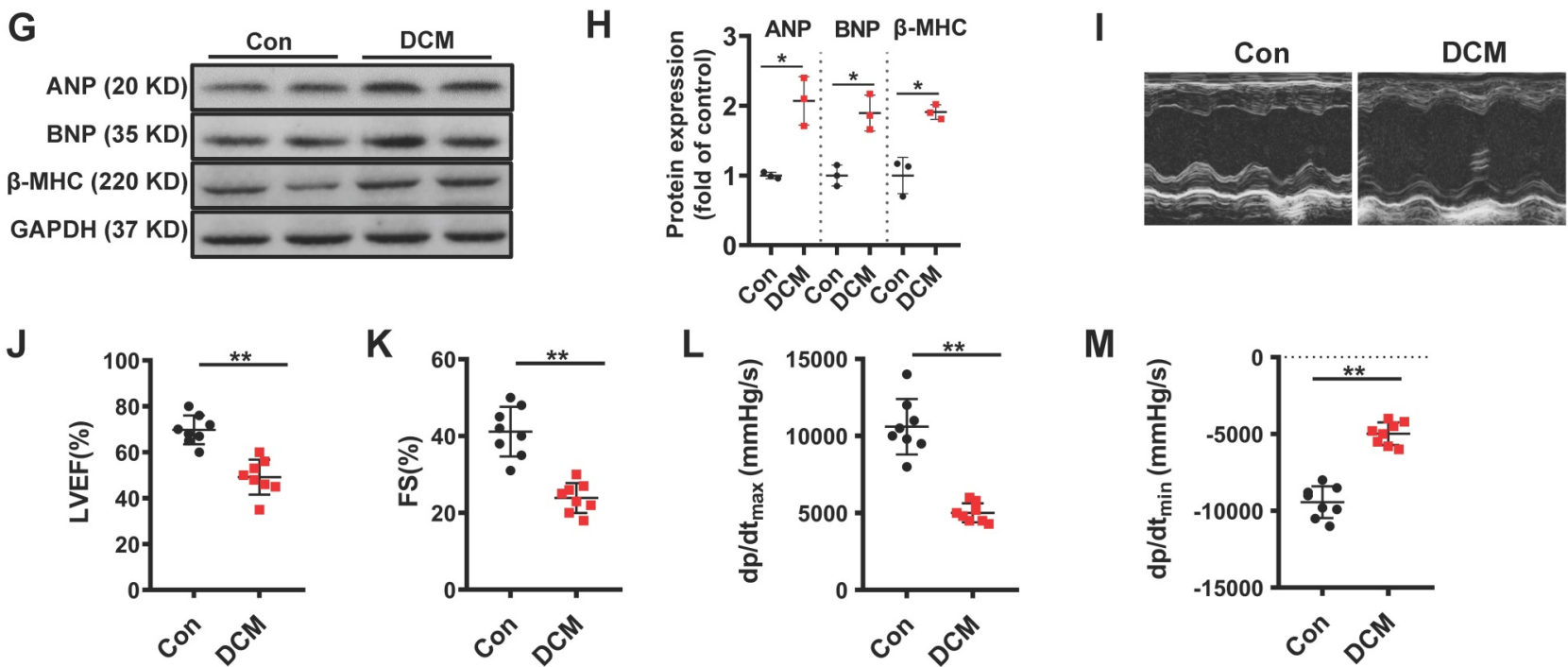

Figure 1. Establishment of diabetic cardiomyopathy mouse model. (A) schematic illustration of interventional studies in mice with STZ-induced hyperglycemia. (B) Line graphs reflecting blood glucose levels in mice with STZ-induced hyperglycemia. Blood glucose was measured at indicated time points. Histological analyses of the H\&E staining and WGA staining of Con and DCM mice 26 weeks after STZ injection. (C) Representative images of H\&E staining in the hearts of Con and DCM mice ( $\mathrm{n} \geq 8$; scale bar, $100 \mu \mathrm{m})$. (D) Statistical results of the myocyte cross-sectional areas ( $\mathrm{n} \geq 6$, at least 100 cells per mouse were analyzed). (E) Representative images of WGA staining in the hearts of Con and DCM mice $(n \geq 8$; scale bar, $100 \mu \mathrm{m})$. (F) Quantitative analysis of myocyte cross-sectional areas ( $\mathrm{n} \geq 6$, at least 100 cells per mouse were analyzed). (G-H) Representative immunoblots of Atrial natriuretic peptide (ANP), B type natriuretic peptide (BNP) and $\beta$-myosin heavy chain (MHC) expression and quantitative analysis are shown. (I-K) Echocardiographic measurements of ventricular functional parameters includes: Ejection fraction (J) and fractional shortening (K). Representative echocardiographic M-mode images (I). (L-M) Cardiac haemodynamic measurements including $+\mathrm{dP} / \mathrm{dt} \mathrm{t}_{\max }$ and $-\mathrm{dP} / \mathrm{dt}_{\min }$ were also conducted. Con, control mice without diabetes, black dots; DCM, diabetic cardiomyopathy, red squares. Data are shown as mean \pm SEM ( $\geq 6$ per group); * $p<0.05$, **p $<0.01$ vs Con group. H\&E, hematoxylin eosin; WGA, wheat germ agglutinin. LVEF, left ventricle ejection fraction; FS, fractional shortening; $+\mathrm{dp}_{\mathrm{d}} / \mathrm{dt}_{\mathrm{max}}$, the maximal rate of pressure development; - dp/dt $t_{\min }$, the minimal rate of pressure decay. 


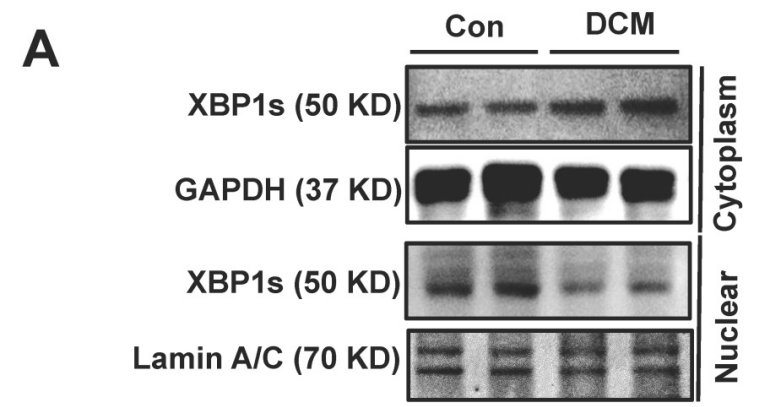

C

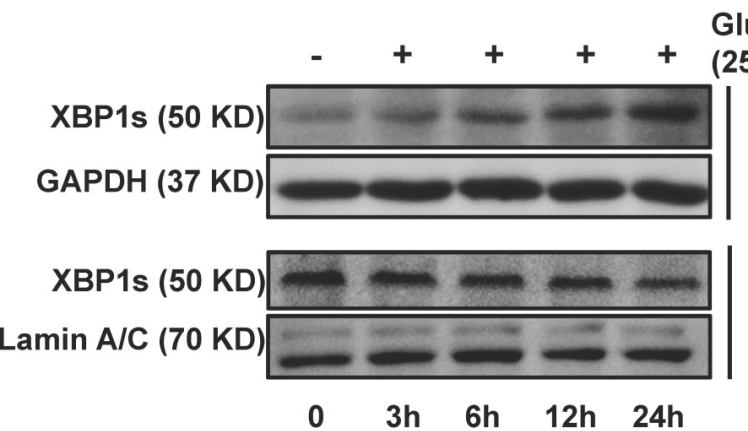

Glucose

B

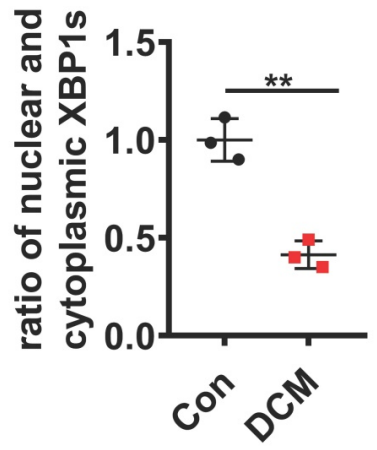

D

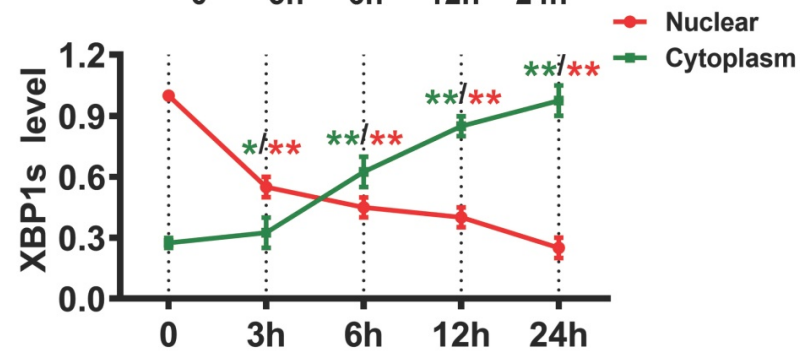

$\mathbf{E}$

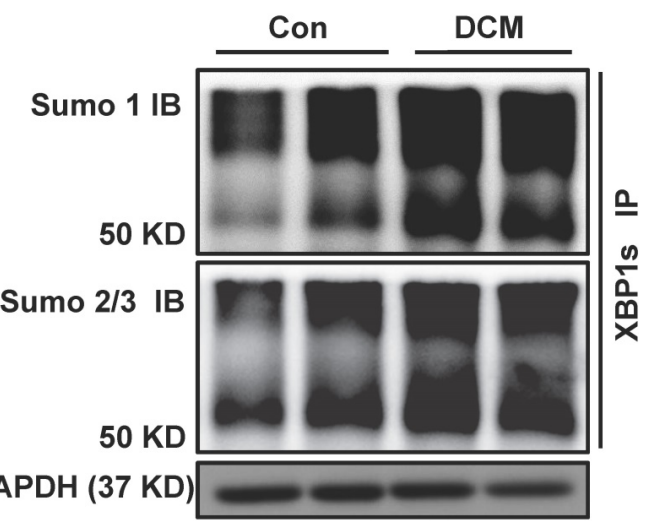

Figure 2. XBPIs' nuclear translocation was significantly reduced and negatively correlated with the level of its SUMOylation in STZ-induced diabetic mouse heart. XBP1s nuclear translocation is impaired in DCM mice and in H9C2 cardiomyocytes treated with HG (25mM). (A-D) Representative immunoblots (A) and bar graph (B) showing nuclear and cytoplasm levels of XBPIs in heart samples of wild-type control (Con) and STZ-induced diabetic cardiomyopathy mice (DCM). As loading controls, Lamin A/C was used for nuclear extracts and GAPDH for cytoplasm extracts ( $\mathrm{n}=6$ mice per group). (C-D) Representative immunoblots showing cytoplasmic (C, top panel) and nuclear levels (C, lower panel) of XBPIs in $\mathrm{H} 9 \mathrm{C} 2$ cardiomyocytes at indicated time points after treatment with $\mathrm{HG}$ (25mM). Line graph (D) reflecting the Mean \pm SEM of three independent experiments. (E) XBPIs' SUMOylation was increased in DCM group. Heart samples from Con and DCM mice were immunoprecipitated with anti-XBPIs antibody, and Western blot analysis was performed with anti-SUMO1 (top panel) and anti-SUMO2/3 (lower panel) antibodies. A significant increase in XBP1s' SUMOylation was observed in DCM group. Con, control mice without diabetes, black dots; DCM, diabetic cardiomyopathy, red squares; IB, immunoblot; IP, immunoprecipitation. Mean \pm SEM, (B, D); ${ }^{* *} p<0.01$ vs Con group. $* p<0.05$ vs 0 h; ** $p<0.01$ vs $0 \mathrm{~h}$ (B: $t$-test; D: ANOVA); Representative immunoblots of at least three independent experiments (A, C and E).

\section{MAPKs are differentially regulated in diabetic mouse heart}

Mitogen-activated protein kinases (MAPKs) consist of three subfamilies, including extracellular signal-regulated kinase 1/2 (ERK1/2), p38, and c-Jun N-terminal kinase (JNK)[32]. A large number of in vivo and in vitro studies have demonstrated that chronic hyperglycemia can activate MAPKs, which play a pivotal role in the development of DCM[33]. To assess MAPK signaling activity, levels of total and phosphorylated ERK1/2, JNK, and p38 in diabetic hearts were determined. We found that the expression of phosphorylated ERK1/2 was significantly increased in diabetic mouse heart when compared to non-diabetic control heart samples. However, the levels of phosphorylated p-JNK and p-p38 were not significantly induced (Figure 4A-B). Therefore, these data indicated ERK1/2 could be one of the important effectors in response to consistent hyperglycemia.

\section{Phosphorylation dependent SUMOylation of XBP Is is triggered by HG-induced ERK $1 / 2$ activation}

Given the specific increase in p-ERK1/2 in the diabetic heart, we next determined whether the phosphorylation of ERK1/2 modulates XBP1s function by modulating its phosphorylation. For this we investigated the ERK1/2 phosphorylation sites on XBP1s. By using the phosphorylation prediction software GPS 3.0 (http://gps.biocuckoo.org/ userguide.php), we identified a series of potential ERK1/2 phosphorylation sites on XBP1s and the three highest-ranking sites (Ser61, Ser169, Ser348; Figure 5A) were selected. We performed site-directed mutagenesis with Alanine (Ala) to analyze the functional relevance of these phosphorylation sites. 


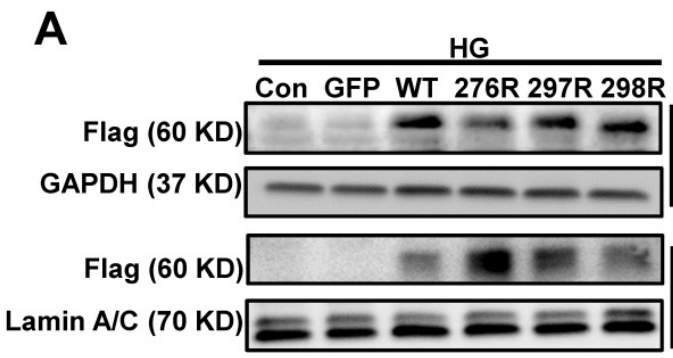

C

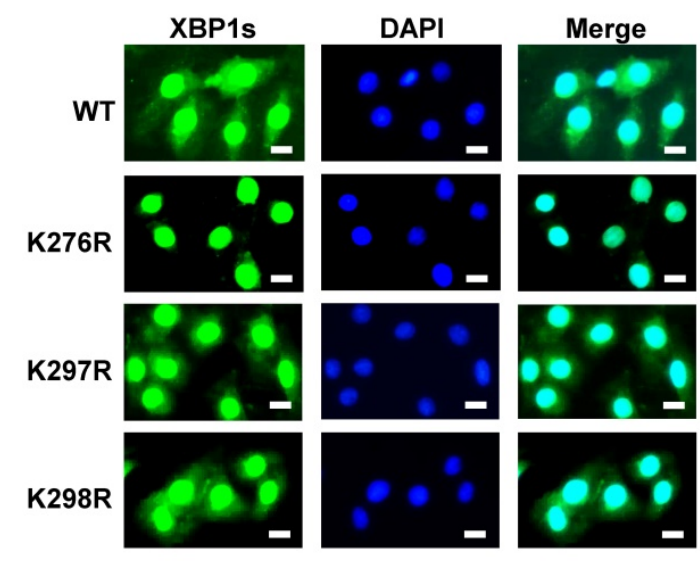

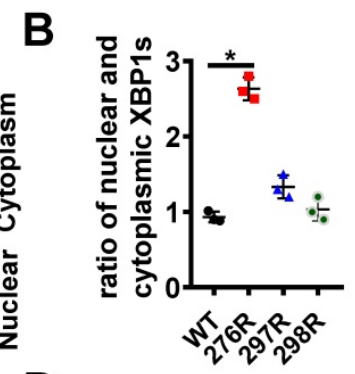

D

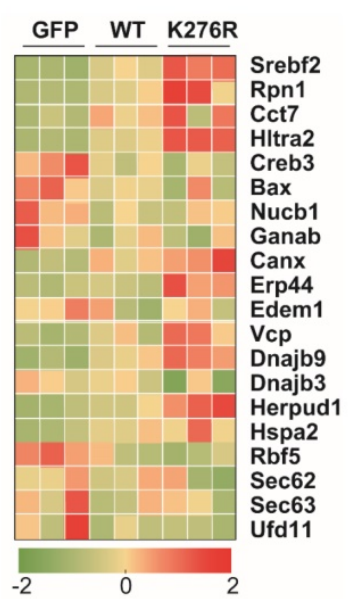

E

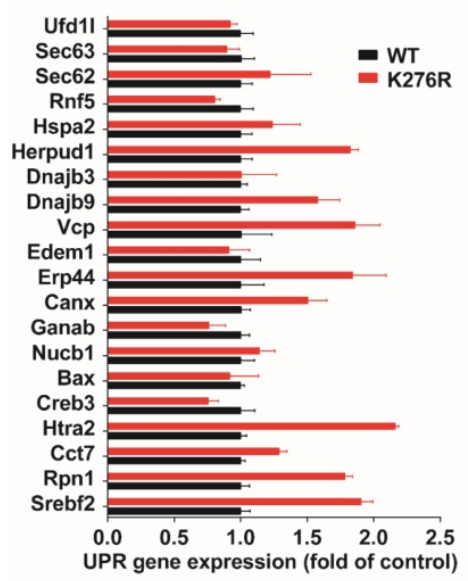

Figure 3. SUMOylation of XBP1s at K276 residue at the C-terminal transactivation domain reduced its nuclear localization under HG condition. (A-B) Immunoblots and bar graph of Flag-XBPIs from Nuclear and cytoplasmic protein extracts in $\mathrm{H} 9 \mathrm{C} 2$ cells upon transfection with different Flag-XBPIs constructs. As loading controls, Lamin A/C was used for nuclear extracts and GAPDH for cytoplasm extracts. (C) Fluorescent microscopic images of XBP1s-GFP fusion constructs upon $24 \mathrm{~h}$ transfection into $\mathrm{H} 9 \mathrm{C} 2$ cells following HG intervention for $6 \mathrm{~h}$. Nuclei were counter-stained with DAPI. Scar bar: $25 \mu \mathrm{m}$. (D) Heat map summarizes enrichment of condition-specific concordant UPR genes. (E) And their impact on regulation of UPR-gene expression (E; $n=3$; Mean \pm SEM, wild type vs K276R mutant).

Phosphorylation is an important protein posttranslational modification[34] that modulates additional post-translational modifications, including ubiquitination, SUMOylation, and acetylation, and in turn nuclear localization of proteins[35]. To determine whether XBP1s phosphorylation regulated its SUMOylation, we transiently transfected HEK293T cells with the Flag-XBP1s-WT and phospho-mutant XBP1s plasmids (S61A, S169A, S348A). The cells were subsequently exposed to HG for 6 hours. Immunoblot results showed that all mutants were functional and equally expressed (Figure 5B). Immunoprecipitation was performed using a Flag antibody. As shown in Figure 5C, the SUMO1 and SUMO2/3 levels were significantly decreased, indicating that mutation of the ERK consensus site (Ser 348) in XBP1s has a substantial effect on XBP1s' phosphorylation and SUMOylation. However, The S69A and S161A mutants showed no decreases in XBP1s SUMOylation (Figure 5C), indicating that the Ser348 site has a crucial role in XBP1s SUMOylation.

To ascertain whether the phosphorylation of $\mathrm{XBP} 1 \mathrm{~s}$ at the Ser348 site by ERK1/2 regulate its SUMOylation in $\mathrm{H} 9 \mathrm{C} 2$ cells, we constructed adenoviruses carrying the XBP1s wild type and S348A mutant and expressed in H9C2 cells. As shown in Figure 5D-E, consistent with the foregoing results, the SUMO1 and SUMO2/3 levels were significantly reduced in S348A mutant in compared with WT construct. These data indicated that ERK1/2 pathway regulates HG-induced XBP1s SUMOylation by phosphorylating Ser348.

\section{ERK1/2 inhibition increases XBP 1 s' nuclear translocation by inhibiting XBP Is' SUMOylation in $\mathrm{H} 9 \mathrm{C} 2$ cells in vitro.}

ERK1/2 signaling pathway could promote cardiac differentiation and plays a role in myocardial protection in myocardial ischemia reperfusion[36, 37]. Additionally, MEK1/2 inhibitor U0126 attenuates ischemia/reperfusion-induced apoptosis and autophagy in myocardium[38] and attenuates cisplatin-induced renal injury by decreasing inflammation and apoptosis by inhibition of ERK1/2 phosphorylation[39]. In agreement with these data, ERK1/2 phosphorylation was increased in diabetic mouse heart (Figure 4A-B) and ERK1/2 pathway regulated HG-induced XBP1s phosphorylation and SUMOylation (Figure 5A-E). So, we used U0126, an inhibitor of MEK1/2, for subsequent experimental studies. 


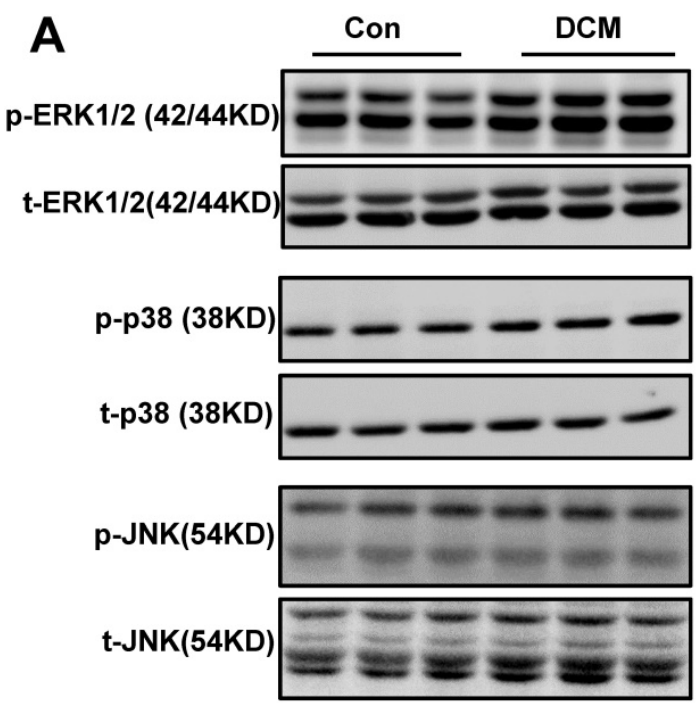

B

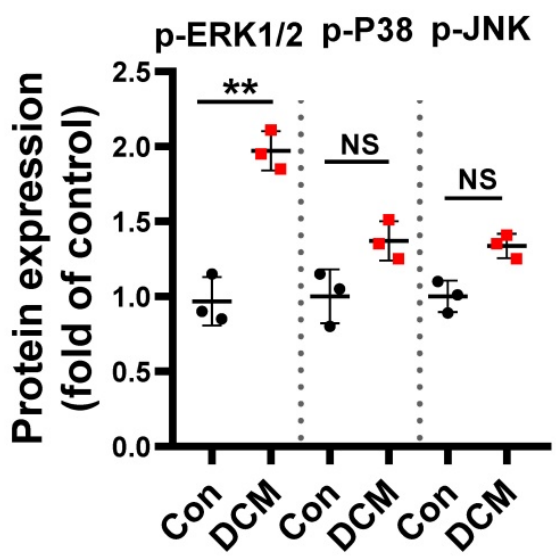

Figure 4. Differential regulation of MAPK kinase in STZ-induced diabetic mouse heart. (A) Western blot analysis of protein extracts from hearts in different mice as indicated using specific antibodies against the phosphorylated and the non-phosphorylated form of the MAPKs ERK1/2, p38, JNK and (B) scatter blot summarized the results. We could show an increase in the phosphorylated form of the MAPKs ERK1/2 but not in p38 and JNK. Con, control mice without diabetes, black dots; DCM, diabetic cardiomyopathy, red squares; Data represent ratios of densitometric measurements of either $\mathrm{p}-\mathrm{JNK}$ or JNK, $\mathrm{p}$-ERK and ERK1/2 or $\mathrm{p}$-p 38 and $\mathrm{p} 38$ and are presented as Mean \pm SEM. **P $<0.01$ vs Con group; NS, non-significant ( $t$-test). Representative immunoblots of at least three independent experiments (A).

A

\begin{tabular}{|c|c|c|c|c|} 
Position & Kinase & Peptide Sequence & Score & Cutoff \\
\hline 61 & CMGC/MAPK/ERK/Erk1 & RQRLTHLSPEEKALR & 19.41 & 17.725 \\
\hline 169 & CMGC/MAPK/ERK/Erk2 & GAGPVVTSPEHLPMD & 12.314 & 10.451 \\
\hline 348 & CMGC/MAPK/ERK/Erk1 & SPFSDMSSPLGTDHS & 23.174 & 17.725 \\
\hline
\end{tabular}

B

\begin{tabular}{llll}
\multicolumn{3}{c}{ HG } \\
\hline GFP & WT & S61A & S169A S348A
\end{tabular}

IB: Flag

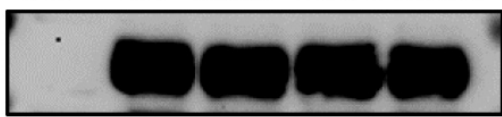

IB: GAPDH

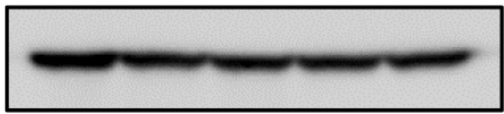

D

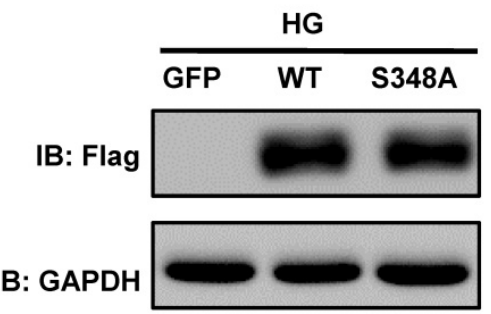

C

HG

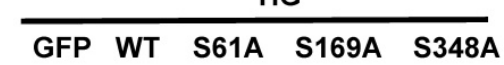

IB: Flag

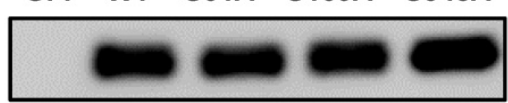

IB: Sumo1

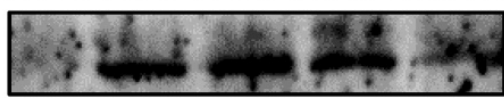

$\frac{\pi}{4}$
$\dot{1}$

IB: Sumo 2/3

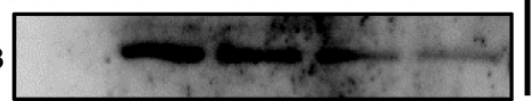

E

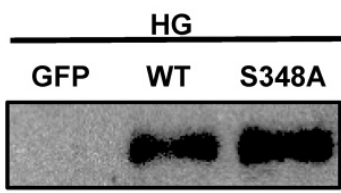

IB: SUMO1

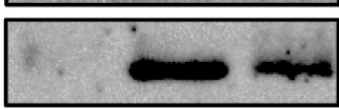

IB: SUMO2/3

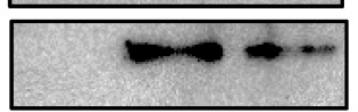

$\frac{\pi}{4}$
$\frac{0}{1}$

Figure 5. Predicting and validating ERK1/2 Serine phosphorylation sites on XBP1s. (A) Potential phosphorylated Serine sites on XBP1s by ERK1/2 were predicted by the phosphorylation prediction software GPS 3.0. (B) Plasmid wild type XBPIs (WT) and serine-to-alanine phospho-mutants of XBPIs (S61A, S169A and S348A) were expressed in HEK293T cells and lysates were immunoblotted using the indicated antibodies. (C)Additionally, lysates were immunoprecipitated (IP) using Flag-agarose and immunoblotted using the indicated antibodies. (D) $\mathrm{H} 9 \mathrm{C} 2$ cells were infected with wild type and XBP1s S348A mutant carrying adenoviruses, lysates were immunoblotted using the indicated antibodies. (E) Subsequently, lysates were immunoprecipitated (IP) using Flag-agarose and immunoblotted using the indicated antibodies. Results are representative of three independent experiments (B-E). 
A
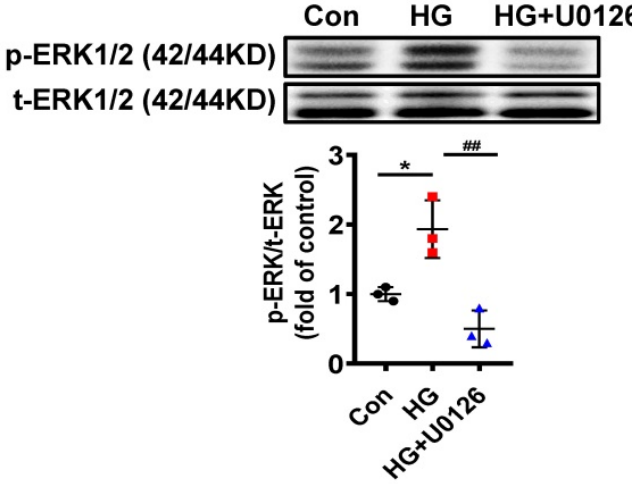

C
B

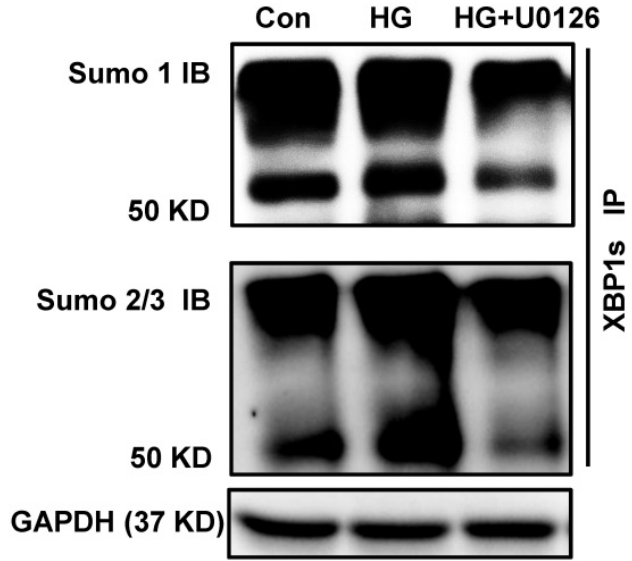

D
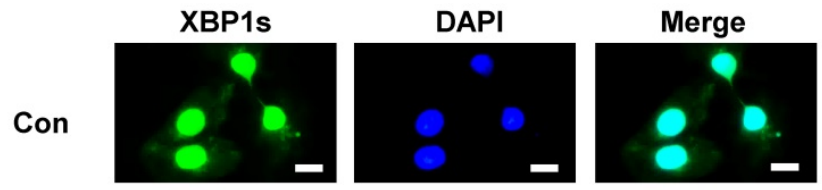

HG
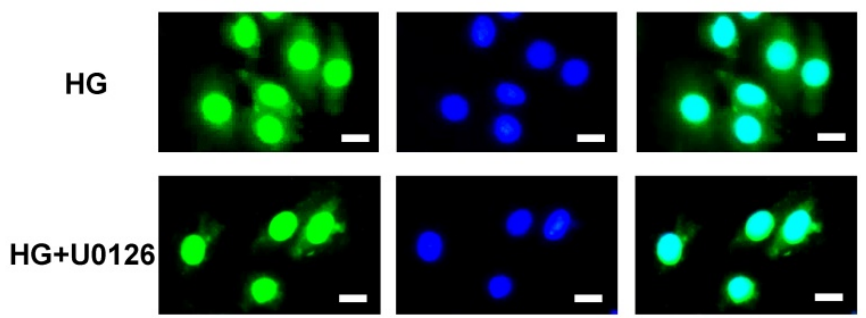

Figure 6. U0126 increased XBP1s' nuclear translocation through inhibiting its SUMOylation in HG-treated H9C2 cells in vitro. (A) Expression of total-ERK1/2 $(\mathrm{t}-\mathrm{ERK} 1 / 2)$ and phospho-ERK1/2 (p-ERK1/2) was detected by Western blot after H9C2 cells pretreated with U0126 (10 $\mu$ M) for $1 \mathrm{~h}$ and HG (25mM) for additional $6 \mathrm{~h}$. Representative immunoblots (top panel) and scatter blot (lower panel) summarizing results. (B) Extracted total proteins were immunoprecipitated with anti-XBPIs antibody, and Western blot analysis was performed with anti-SUMO1 (top panel) and anti-SUMO2/3 (lower panel) antibodies. A significant increase in XBPIs-SUMOylation was observed in HG group. (C) Representative immunoblots (top panel) and scatter blot (lower panel) showing cytoplasm and nuclear levels of XBPIs in the extracted total proteins of Con, HG and HG + U0126 group. As loading controls, Lamin A/C was used for nuclear extracts and GAPDH for cytoplasm extracts. (D) In the same experimental setting immunofluorescence staining was conducted, $\mathrm{XBP} 1 \mathrm{~s}$ was stained with green color, while nuclei were counter stained with DAPI (blue color). Scar bar: $25 \mu \mathrm{m}$. Con, control without HG, black dots; HG: high glucose, squares; U0126, blue triangles; IB, immunoblot; IP, immunoprecipitation. Mean \pm SEM (A, B); *P < 0.05 vs Con group, \#P < 0.01 vs HG group (A, B: ANOVA); Representative IBs of at least three independent experiments (A, B and C).

We initially determined the efficacy of U0126 on the phosphorylation of ERK1/2 (p-ERK1/2). H9C2 cells pretreated with $10 \mu \mathrm{M}$ U0126 for 1 hour were incubated with HG $(25 \mathrm{mM})$ for additional 6 hours. As shown in Figure 6A, U0126 markedly reduced p-ERK1/2 level induced by HG.

To determine if the reduction of $\mathrm{p}$-ERK $1 / 2$ by U0126 can regulate XBP1s' SUMOylation, we initially determined XBP1s SUMOylation in $\mathrm{H} 9 \mathrm{C} 2$ cells in response to U0126 treatment. The results demonstrate that U0126 significantly inhibit XBP1 SUMOylation induced by HG in H9C2 cells (Figure 6B). These data suggest that XBP1s SUMOylation is dependent on ERK phosphorylation in vitro.

Similar to increasing XBP1s' SUMOylation, U0126 promotes XBP1s' nuclear translocation, which is inhibited by HG in H9C2 cells (Figure 6C-D). Taken together, ERK1/2 inhibition with U0126 can increase $\mathrm{XBP1s^{ \prime }}$ nuclear translocation by inhibiting XBP1s'
SUMOylation in HG-induced H9C2 cells in vitro.

\section{ERK1/2 inhibition protects against DCM in mouse model of type 1 diabetes}

In order to confirm whether U0126 could ameliorate diabetic cardiomyopathy, we conducted animal experiments in a well-established mouse model of streptozotocin (STZ)-induced type 1 diabetes (Figure 7A). 18 weeks after persistent hyperglycemia, 8 weeks U0126 $(1 \mathrm{mg} / \mathrm{kg})$ or vehicle administration did not alter blood glucose in DCM mice (Figure 7B). To investigate the possible protective effects of U0126 on cardiac hypertrophy, we conducted Hematoxylin and eosin (H\&E) staining and WGA staining of heart tissue. We found that aberrant structural abnormalities, including disrupted cardiac fibers, deranged cellular structures, obscured intercellular border and increased cardiomyocyte transverse cross-sectional areas in diabetic model 
group (Figure 7C-F). U0126 treatment significantly ameliorated the structural abnormalities in the hearts of diabetic mice. Consistent with the morphologic observations, the protein expression, markers of cardiac hypertrophy, were significantly increased in diabetic hearts, which were also reversed by U0126 treatment (Figure 7G-H).

Before sacrifice, we evaluated the effects of U0126 on the cardiac function. Left ventricular performance was assessed by echocardiography. Compared to vehicle treated diabetic mice, mice treated with U0126 showed a significant reduction of fraction shorting and ejection fraction (Figure 7I-K). We also detected the left ventricular hemodynamic alternation after administering. The result showed that diabetic mice has aggravated cardiac dysfunction, U0126 treatment decreased $+\mathrm{dP} / \mathrm{dt} \max$ and increased $-\mathrm{dP} / \mathrm{dt}_{\min }$ (Figure $\left.7 \mathrm{~L}-\mathbf{M}\right)$. These data demonstrated U0126 administration exerted the beneficial effect on the left ventricle function in diabetic mice, even at a late stage.

\section{U0126 inhibits XBP 1s' SUMOylation and increases XBP Is' nuclear translocation in diabetic mouse heart in vivo}

To determine whether U0126 ameliorates diabetic cardiomyopathy by inhibiting XBP1s SUMOylation and increasing XBP1s nuclear translocation in vivo, we also determined the efficacy of U0126 on the phosphorylation of ERK1/2 in DCM mice. Consistent with the protective effects in H9C2 cells p-ERK1/2 was induced in STZ-induced in mice with DCM and U0126 markedly reversed this process (Figure 8A).
A

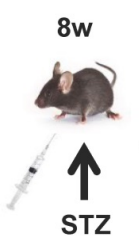

B

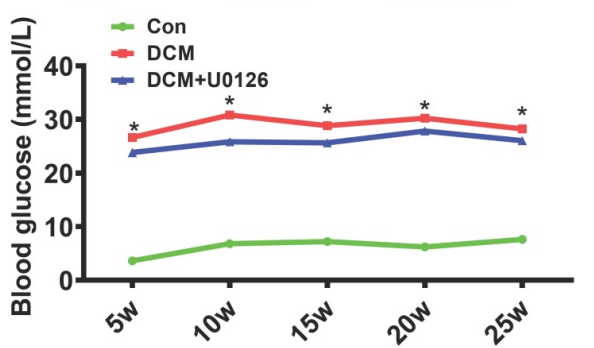

G

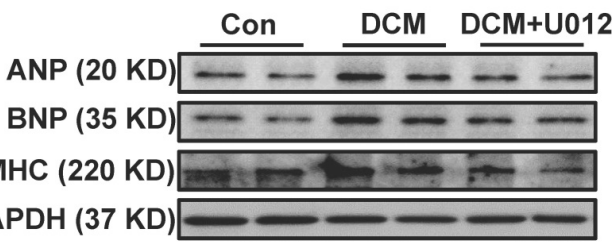

ANP BNP $\beta-M H C$
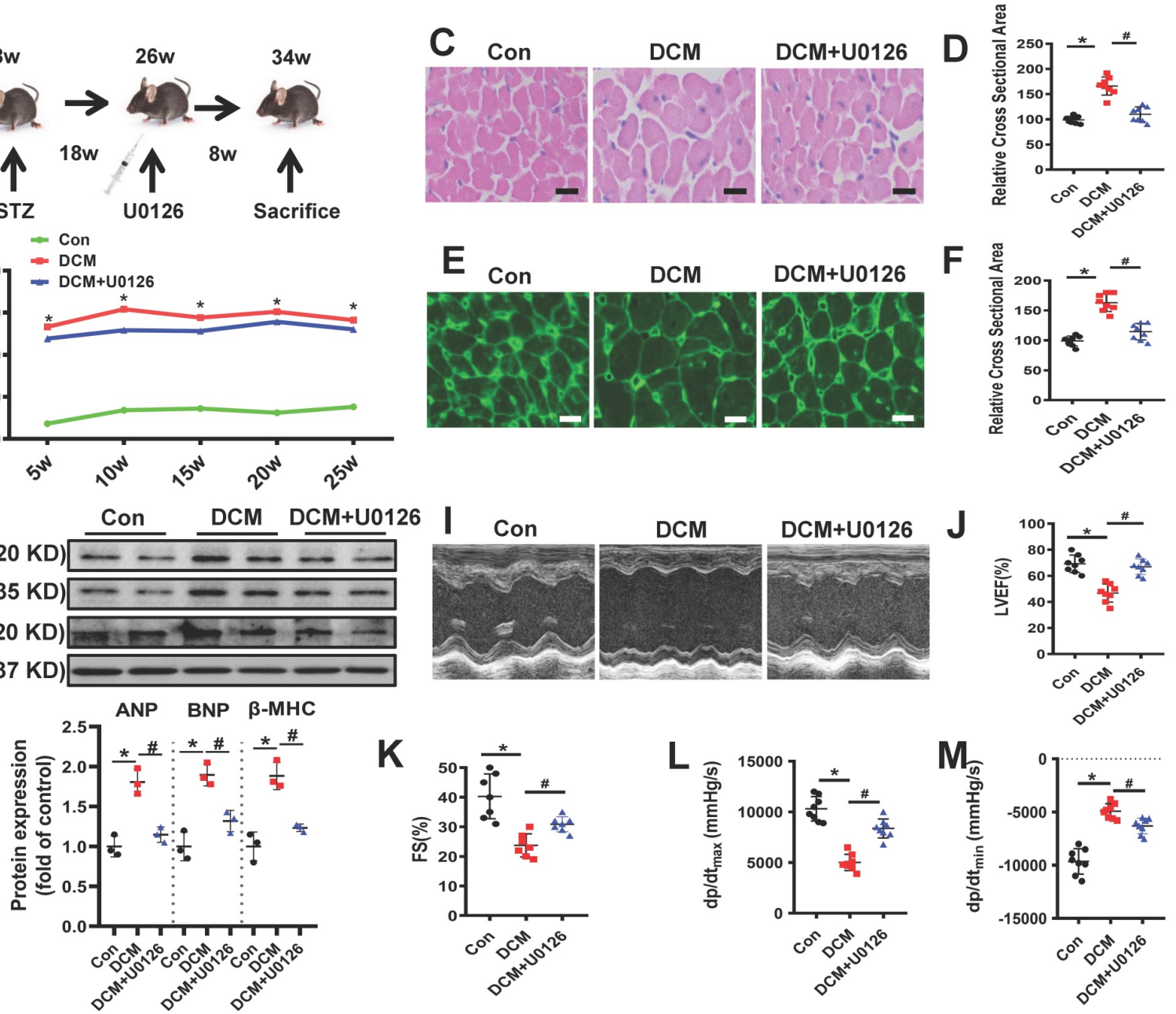

K

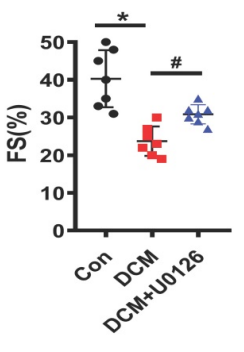

L

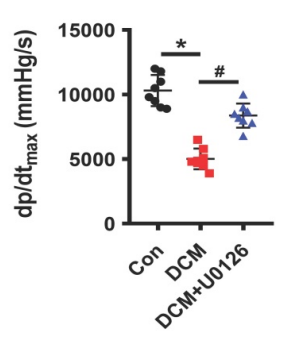

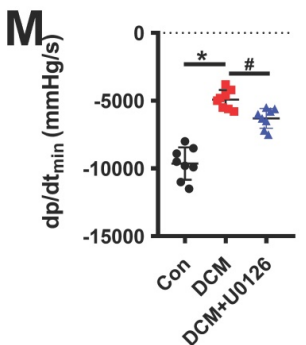

Figure 7. U0126 improved cardiac function and ameliorated cardiac hypertrophy in STZ-induced diabetic mice. (A) Schematic illustration of interventional studies in mice with STZ-induced hyperglycemia. Treatment with U0126 for 8 weeks was initiated after manifestation of abnormal cardiac function at week 18 . (B) Line graphs reflecting blood glucose levels in mice with STZ-induced hyperglycemia. Blood glucose was measured at indicated time points. Histological analyses of the H\&E staining and WGA staining of hearts from different mice as indicated 26 weeks after STZ injection. (C) Representative images of H\&E staining in the hearts of each group mice ( $\mathrm{n} \geq 8$; scale bar, $100 \mu \mathrm{m})$. (D) Statistical results of the myocyte cross-sectional areas ( $\mathrm{n} \geq 6$, at least 100 cells per mouse were analyzed). (E) Representative images of WGA staining in the hearts of each group mice $(n \geq 8$; scale bar, $100 \mu \mathrm{m})$. (F) Quantitative analysis of myocyte cross-sectional areas $(n \geq 6$, at least 100 cells per mouse were analyzed). Representative immunoblots (G) and scatter blots summarizing results $(\mathbf{H})$ for cardiac hypertrophy-related proteins expression in hearts from different mice as indicated. (I) Representative echocardiographic images of the left ventricle in different mice as indicated; (J-K) Echocardiographic assessment of LVEF and FS in different mice as indicated. (L-M) Cardiac hemodynamic measurements including $+\mathrm{dP} / \mathrm{dt}_{\max }$ and $-\mathrm{dP} / \mathrm{dt}_{\mathrm{min}}$ were also conducted. Con, control mice without diabetes, black dots; DCM, diabetic cardiomyopathy, red squares; U0126 1mg/kg, blue triangles; Data are shown as Mean \pm SEM; $* p<0.05$ vs Con group; $\#<0.05$ vs DCM group. H\&E, hematoxylin eosin; WGA, wheat germ agglutinin. LVEF, left ventricle ejection fraction; FS, fractional shortening; $+\mathrm{dp}_{\mathrm{d}} / \mathrm{dt}_{\max }$, the maximal rate of pressure development; - $\mathrm{dp} / \mathrm{dt}$ min, the minimal rate of pressure decay; Representative IBs of at least three independent experiments $(\mathrm{G})$. 
A

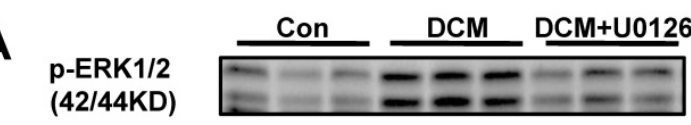

C

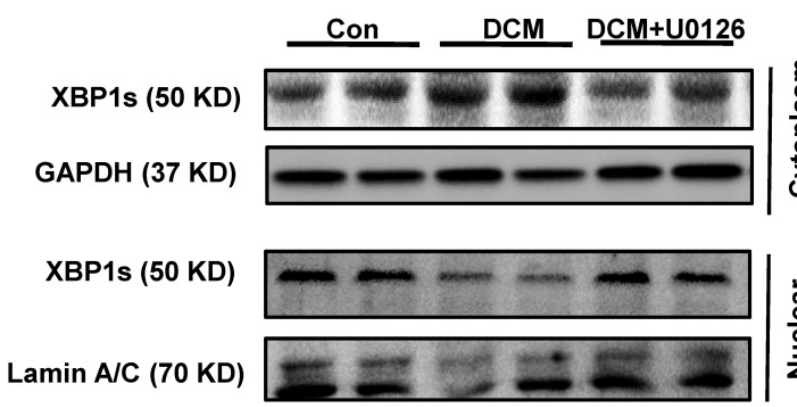

B Con DCM DCM+U0126

Sumo 1 IB

$50 \mathrm{KD}$

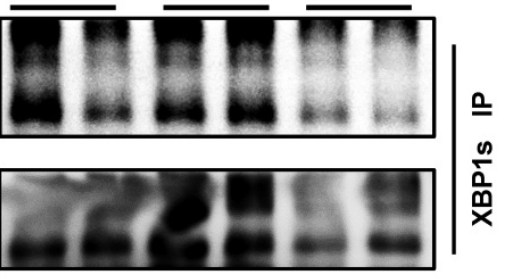

GAPDH (37 KD) $-\infty-\infty$

D

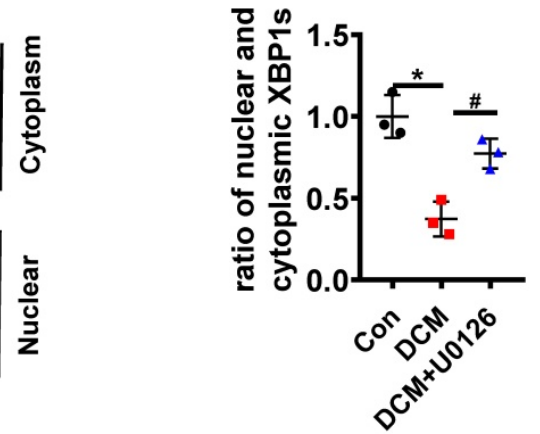

Figure 8. U0126 increased XBP Is' nuclear translocation through inhibiting its SUMOylation in STZ-induced diabetic mouse heart in vivo. Heart samples of each group were lysed and the extracted total proteins were processed for the detection of p-ERK1/2 and t-ERK1/2 using Western blot. (A) Representative immunoblots (top panel) and scatter blots (lower panel) summarizing results. (B) The extracted total proteins of heart samples were also immunoprecipitated with anti-XBPIs antibody, and Western blot analysis was performed with anti-SUMO1 (top panel) and anti-SUMO2/3 (lower panel) antibodies. (C) Representative immunoblots and (D) scatter blots showing nuclear and cytoplasm levels of XBPIs in the extracted proteins of each group mice. As loading controls, Lamin A/C was used for nuclear extracts and GAPDH for cytoplasm extracts. Con, control without HG, black dots; DCM: Diabetic cardiomyopathy, red squares; U0126 $1 \mathrm{mg} / \mathrm{kg}$, blue triangles; Data are shown as mean \pm SEM; $* p<0.05$ vs Con group; $\#$ p 0.05 vs DCM group. IB, immunoblot; IP, immunoprecipitation. Representative IBs of at least three independent experiments (A, B and C).

Additionally, in congruent with the protective effect of U0126 in H9C2 cells in vitro, U0126 significantly attenuated XBP1s' SUMOylation induced by hyperglycemia in DCM mice (Figure 8B). Likewise, U0126 treatment restored XBP1s' activity by promoting its nuclear translocation (Figure 8C-D).

Taken together, our results identified the crucial role of ERK1/2 pathway in DCM. Chronic hyperglycemia activates Ras/MEK/ERK cascades, leading to XBP1s' Ser 348 phosphorylation, subsequently resulting in XBP1s' K276 SUMOylation and finally restricting its nuclear translocation and activity. U0126 could ameliorates DCM by inhibiting Ras/MEK/ERK cascades to maintain XBP1s' nuclear translocation (Figure 9).

\section{Discussion}

Effective therapeutic approaches are urgently needed to improve clinical outcomes of patients with DCM. However, despite intense research, the pro-pathogenic factors that can be modulated to prevent DCM remain poorly understood. In particular, the involvement of dysregulated proteostasis as result of ER stress in this process remains obscure. Our studies using a STZ induced mouse model of T1DM in vivo and complementary experiments in $\mathrm{H} 9 \mathrm{C} 2$ cardiomyoblasts in vitro provide the following major findings: 1 . XBP1s' nuclear translocation in cardiomyocyte correlates well with the DCM outcomes; 2. Chronic hyperglycemia induced activation of MAP kinase ERK1/2 can phosphorylate XBP1s on its serine residue 348, which requires for its SUMOylation on lysine residue 276 and results in its cytoplasmic retention; 3. ERK1/2 inhibitor U0126 can promote XBP1s' nuclear translocation in cardiomyocyte by restricting its phosphorylation and SUMOylation in vitro and in vivo, provides novel therapeutic avenue for DCM.

Studies showed that HF and case-fatality remains higher in T1DM than in people without diabetes mellitus, moreover T1DM patients have an even higher incidence of HF than T2DM[9, 10, 40]. Since clinical features differ between the two types of $\mathrm{DM}$, underlying mechanism of DCM may be partially distinct[11]. Consistent with the hypothesis, new evidences are emerging. For instance, experimentally 


\section{Cardiomyocyte}

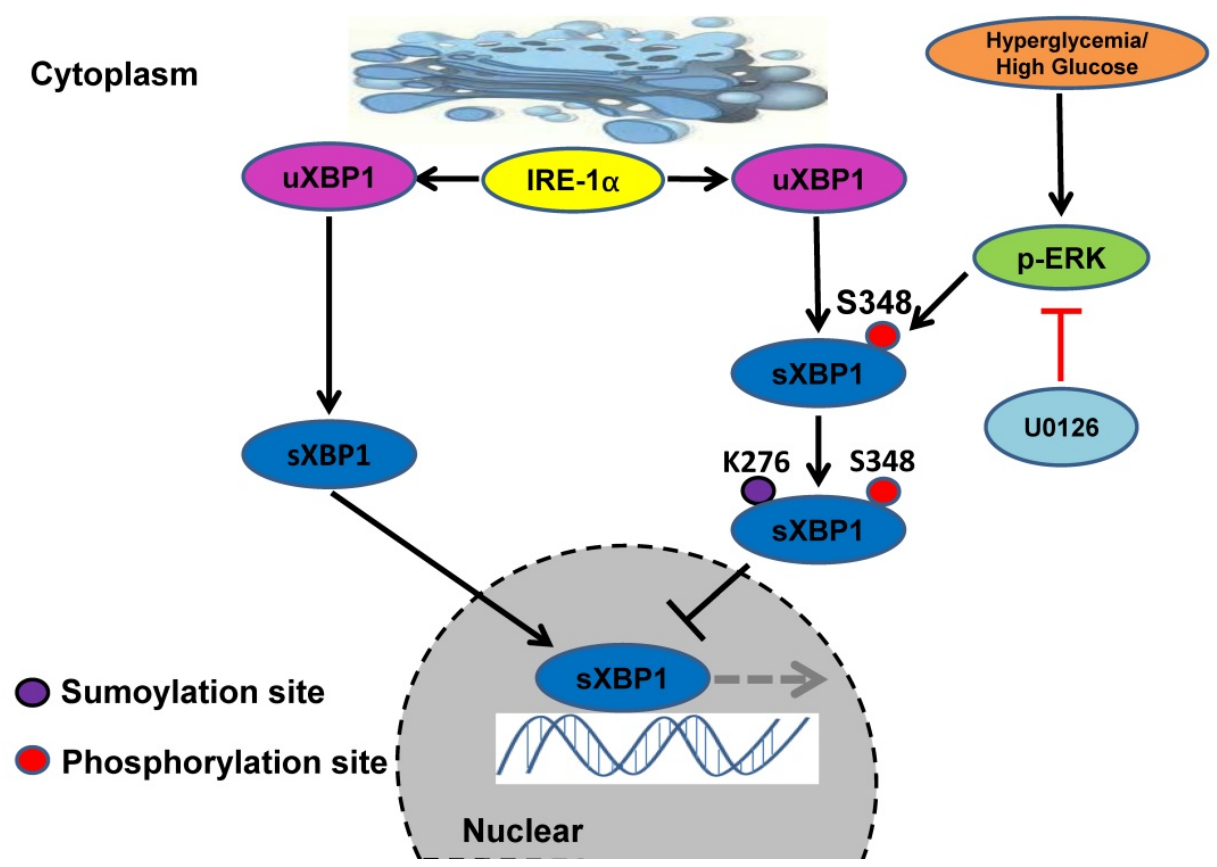

Figure 9. Schematic model of the signaling pathway for XBPIs' nuclear translocation regulated by U0126 through restricting its phosphorylation and SUMOylation. In healthy subjects the XBPIs' nuclear translocation maintains normal level. In contrast, in diabetic individuals, hyperglycemia activates Ras/MEK/ERK cascades, these activation signals lead to XBP1s Ser 348 phosphorylation, resulting in XBPIs K276 SUMOylation and subsequent impairing its nuclear translocation. U0126 could ameliorates diabetic cardiomyopathy by inhibiting Ras/MEK/ERK cascades to maintain XBPIs' nuclear translocation.

cardiomyocyte autophagy was enhanced in T1DM but suppressed in T2DM mouse models[41]. Clinically neither ACE-inhibitor nor statin therapy worked effectively in T1DM patients when compared to T2DM patients[42]. Therefore, DCM in T1DM is distinct from the DCM in T2DM and requires separate assessment[13]. Nevertheless, nowadays diabetes appears to be largely used as a synonym for T2DM[14]. Thus, it is prerequisite to explore the unique pathophysiological mechanisms of DCM in T1DM. Within the current study we focused on the DCM in a STZ induced T1DM mouse model and found that the key transcription factor of mammalian UPR XBP1s plays a pivotal role. In consistent with our findings XBP1s has been reported to regulate heart function in ischemia reperfusion Injury and isoproterenol-induced hypertrophy mouse models[43, 44].

Diabetic cardiomyopathy is initially characterized by myocardial fibrosis, dysfunctional remodeling, and associated diastolic dysfunction, later by systolic dysfunction, and eventually by clinical heart failure[4]. In order to unveil the underlying mechanisms for this cardiac complication, many DCM animal models have been developed[45-47]. However, there is so far no standard protocol for establishing a T1DM DCM rodent model. Regarding STZ induced T1DM rodent model, investigators use different dosages and time points, the later range from 4 to 24 weeks[48-50]. Intriguingly, Gu et. al. analyzed STZ induced T1DM rat model at different time points, including 2, 12 and 24 weeks. Echocardiography analysis revealed that cardiac dysfunction is observed at 12 weeks onwards, while cardiac fibrosis and hypertrophy only became obvious at 24-week time point[50]. Albuminuria is a marker of microangiopathy and endothelial dysfunction and high level of albuminuria is associated with left ventricular dysfunction, especially in asymptomatic diabetic patients[51, 52]. Our previous study showed that in a T1DM diabetic nephropathy mouse model, a reduction in nuclear XBP1s associated with increased nuclear ATF6 and CHOP in kidney was apparent at 10 weeks and peaked at 26 weeks post STZ injection and coincided with the onset of albuminuria. When the hyperglycemia and albuminuria were stably induced, the mice were treated with the chemical chaperone TUDCA at 18 weeks post STZ injection to attenuate ER stress. Surprisingly, after 8 weeks treatment we observed an increase in nuclear XBP1s associated with decreased nuclear ATF6 and CHOP in kidney and alleviated albuminuria, reflecting partial disease reversal[20]. Based on these observations, we did the intervention at 18 weeks and the final analysis at 26 weeks post STZ injection in the current study. While a recent study demonstrates that STZ induced diabetic female mice exhibit a heightened susceptibility to 
diastolic dysfunction, despite exhibiting a lower extent of hyperglycemia than male mice[53], we used only male mice throughout the study to avoid the gender discrepancy.

As mentioned above, we observed a positive correlation between HF indices and nuclear translocation of XBP1s in T1DM mouse hearts in vivo and high glucose treated $\mathrm{H} 9 \mathrm{C} 2$ cardiomyoblasts in vitro. Contrarily, Wang et al. reported recently that in cultured H9C2 cardiomyoblasts high glucose promoted the nuclear translocation of XBP1s and CHOP expression, resulting in cardiomyocytes apoptosis in vitro[54]. However, we and others could show that high glucose impaired the nuclear localization of XBP1s in cultured renal epithelial cells, endothelial cells and mesangial cells in vitro and in kidney of diabetic animals in vivo as well[53, 55, 56]. Moreover, cardiomyocyte-specific overexpression of $\mathrm{XBP1s}$ in mice protects against ischemia-reperfusioninduced injury whereas silencing XBP1s in the heart exacerbated the injury in a hexosamine biosynthesis dependent pathway[43]. These studies indicate a protective role of nuclear XBP1s accumulation in cardiac injury and diabetic nephropathy. Besides, in mouse models of obesity nuclear translocation of $\mathrm{XBP1s}$ in hepatocytes could improve the glucose metabolism [57, 58].

Cross-talks between various protein posttranslational modifications are critical for the regulation of coordinated networks for physiological functions [59, 60]. In the current study we could show that S348 phosphorylation of XBP1s is required for its K276 SUMOylation and nuclear exportation under high glucose condition in cardiomyocytes. Our results are in agreement with findings on other transcriptional factors, such as GATA-1, heat shock factors and MEF2A. Paradoxically, SUMOylation can be impaired by phosphorylation modification. For instance, MAPK can block the SUMOylation of AIB1 protein via its phosphorylation[59]. Moreover, there is evidence on SUMOylation dependent phosphorylation and as well[61]. As an example, the SUMOylation status of a subunit of casein kinase II can affect the phosphorylation of its substrates[59]. Interestingly, Hietakangas $\mathrm{V}$ et al. discovered a phosphorylation-dependent SUMOylation motif, which is composed of a SUMOylation consensus site and an adjacent proline-

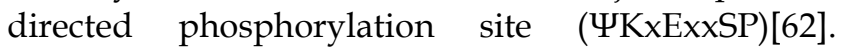
However, such motif does not exist in XBP1s, implying the existence of other possible motifs. Transcription factors SUMOylation can regulate in the intracellular localization of target proteins[63, 64]. Our data suggested an inhibitory effect of K276 SUMOylation on XBP1s' nuclear translocation, since a loss of function mutant K276R promoted its nuclear translocation in cardiomyocytes under high glucose condition. Future studies with cardiomyocyte specific XBP1 SUMOylation and phosphorylation mutants/knock-in mice models are required for further verification of the pathophysiological mechanisms of these post-translational modifications in vivo. In general, the complexity of the posttranslational modification cross-talks contributes to the fine tuning of the physiological and pathological proteomic network regulation and it necessitates further elucidation.

Numerous kinases, such as MAPKs, CKD5, AKT, IKKbeta, etc., have been identified to regulate the phosphorylation status of $\mathrm{XBP} 1 \mathrm{~s}[65-68]$. In the current study, we found ERK1/2, a member of the MAPK family was specifically regulated in the diabetic mouse heart samples. Thus, using in-silico analysis, we obtained the potential phosphosites of ERK on XBP1s and further site directed mutagenesis study identified that S384 was crucial for its SUMOylation modification[66]. Subsequent ERK inhibitor U0126 intervention ameliorated DCM indices in mice, which implies that inhibiting phosphorylation of XBP1s could be one of the possible mechanisms. Since ERK has much diverse cellular roles and its inhibitor has potential off-target effects[69], we could not exclude other possibilities. Hence, cardiomyocyte specific XBP1 SUMOylation and phosphorylation mutants knock in mice models would be better approaches in the future.

\section{Conclusion}

Taken together, we have confirmed the previously unknown function of MEK/ERK pathway in regulation of ER-stress in DCM, and that was related to XBP1s' posttranslational modification cross-talks in cardiomyocyte. Targeting MEK/XBP1 pathway could be a therapeutic avenue for the treatment of DCM.

\section{Abbreviations}

DCM: diabetic cardiomyopathy; CVD: cardiovascular disease; ER-Stress: endoplasmic reticulum stress; STZ: streptozotocin; XBP1: X-box binding protein; HF: heart failure; UPR: unfolded protein response; SENP1: Sentrin/SUMO-specific protease 1; WGA: wheat germ agglutinin; ANP: atrial natriuretic peptide; BNP: B type natriuretic peptide; $\beta$-MHC: $\quad \beta$-myosin heavy chain; MAPKs: Mitogen-activated protein kinases; ERK1/2: extracellular signal-regulated kinase 1/2.

\section{Supplementary Material}

Supplementary figures and table.

http://www.ijbs.com/v17p2984s1.pdf 


\section{Acknowledgements}

We thank Dr. Ling Qi (Cornell University) for providing plasmids encoding wildtype XBP1s and lysine mutants (K276R, K297R and K298R). We thank Zheng Wen and Chen Chen for their valuable technical support. This work was presented as a poster at the annual AHA (American Heart Association) meeting in Chicago, IL, USA, Nov. 2018.

\section{Funding}

The project was supported by the National Natural Science Foundation of China (No.81600301, No.81770680, No. 81873523 and No. 81800411) to HW, WD, HZ and $\mathrm{XH}$, the Key Project of Health and Family Planning Commission of Hubei Province, China (No.WJ2017Z012) to HZ, the Wuhan Morning Light Plan of Youth Science and Technology (No.2016070204010135) to HW, Medicine and Health Science Technology Development Program of Shandong Province (No.2018WS050) to TW.

\section{Consent for publication}

All authors gave their consent for publication.

\section{Availability of data and materials}

Additional data will be made available to all readers upon request per email.

\section{Author Contributions}

TW, JW and WD designed the study, analysed and interpreted the data, and drafted the paper. MW, $X Z, W Z, L D, Y X, Y L, X H, W L$ and TM contributed to data acquisition. HZ and HW designed the study and drafted the paper. All authors read and approved the final manuscript.

\section{Competing Interests}

The authors have declared that no competing interest exists.

\section{References}

1. Rawshani A, Rawshani A, Franzen S, Sattar N, Eliasson B, Svensson AM, et al. Risk Factors, Mortality, and Cardiovascular Outcomes in Patients with Type 2 Diabetes. The New England journal of medicine. 2018; 379: 633-44.

2. Group ASC, Bowman L, Mafham M, Wallendszus K, Stevens W, Buck G, et al. Effects of Aspirin for Primary Prevention in Persons with Diabetes Mellitus. The New England journal of medicine. 2018; 379: 1529-39.

3. Rubler S, Dlugash J, Yuceoglu YZ, Kumral T, Branwood AW, Grishman A. New type of cardiomyopathy associated with diabetic glomerulosclerosis. The American journal of cardiology. 1972; 30: 595-602.

4. Jia G, Hill MA, Sowers JR. Diabetic Cardiomyopathy: An Update of Mechanisms Contributing to This Clinical Entity. Circulation research. 2018; 122: 624-38.

5. DiMeglio LA, Evans-Molina C, Oram RA. Type 1 diabetes. Lancet. 2018; 391: 2449-62.

6. Rawshani A, Sattar N, Franzen S, Rawshani A, Hattersley AT, Svensson AM, et al. Excess mortality and cardiovascular disease in young adults with type 1 diabetes in relation to age at onset: a nationwide, register-based cohort study. Lancet. 2018; 392: 477-86.

7. Miller RG, Costacou T, Orchard TJ. Risk Factor Modeling for Cardiovascular Disease in Type 1 Diabetes in the Pittsburgh Epidemiology of Diabetes Complications (EDC) Study: A Comparison With the Diabetes Control and
Complications Trial/Epidemiology of Diabetes Interventions and Complications Study (DCCT/EDIC). Diabetes. 2019; 68: 409-19.

8. Petrie JR, Sattar N. Excess Cardiovascular Risk in Type 1 Diabetes Mellitus. Circulation. 2019; 139: 744-7.

9. McAllister DA, Read SH, Kerssens J, Livingstone S, McGurnaghan S, Jhund P, et al. Incidence of Hospitalization for Heart Failure and Case-Fatality Among 3.25 Million People With and Without Diabetes Mellitus. Circulation. 2018; 138: 2774-86.

10. Lee YB, Han K, Kim B, Lee SE, Jun JE, Ahn J, et al. Risk of early mortality and cardiovascular disease in type 1 diabetes: a comparison with type 2 diabetes, a nationwide study. Cardiovascular diabetology. 2019; 18: 157.

11. Holscher ME, Bode C, Bugger H. Diabetic Cardiomyopathy: Does the Type of Diabetes Matter? International journal of molecular sciences. 2016; 17.

12. Sousa GR, Pober D, Galderisi A, Lv H, Yu L, Pereira AC, et al. Glycemic Control, Cardiac Autoimmunity, and Long-Term Risk of Cardiovascular Disease in Type 1 Diabetes Mellitus. Circulation. 2019; 139: 730-43.

13. Iso T, Takahashi K, Yazaki K, Ifuku M, Nii M, Fukae T, et al. In-Depth Insight Into the Mechanisms of Cardiac Dysfunction in Patients With Type 1 Diabetes Mellitus Using Layer-Specific Strain Analysis. Circulation journal : official journal of the Japanese Circulation Society. 2019; 83: 1330-7.

14. Mamet H, Petrie MC, Rocchiccioli P. Type 1 diabetes mellitus and coronary revascularization. Cardiovascular endocrinology \& metabolism. 2019; 8: 35-8.

15. Yilmaz S, Canpolat U, Aydogdu S, Abboud HE. Diabetic Cardiomyopathy; Summary of 41 Years. Korean circulation journal. 2015; 45: 266-72.

16. Li J, Zhu H, Shen E, Wan L, Arnold JM, Peng T. Deficiency of rac1 blocks NADPH oxidase activation, inhibits endoplasmic reticulum stress, and reduces myocardial remodeling in a mouse model of type 1 diabetes. Diabetes. 2010; 59: 2033-42.

17. Yang L, Zhao D, Ren J, Yang J. Endoplasmic reticulum stress and protein quality control in diabetic cardiomyopathy. Biochimica et biophysica acta. 2015; 1852: 209-18.

18. Lee AH, Heidtman K, Hotamisligil GS, Glimcher LH. Dual and opposing roles of the unfolded protein response regulated by IRE1alpha and XBP1 in proinsulin processing and insulin secretion. Proceedings of the National Academy of Sciences of the United States of America. 2011; 108: 8885-90.

19. Liu J, Liu Y, Chen L, Wang Y, Li J. Glucagon-Like Peptide-1 Analog Liraglutide Protects against Diabetic Cardiomyopathy by the Inhibition of the Endoplasmic Reticulum Stress Pathway. Journal of diabetes research. 2013; 2013: 630537.

20. Madhusudhan T, Wang H, Dong W, Ghosh S, Bock F, Thangapandi VR, et al. Defective podocyte insulin signalling through p85-XBP1 promotes ATF6-dependent maladaptive ER-stress response in diabetic nephropathy. Nature communications. 2015; 6: 6496.

21. Chen H, Qi L. SUMO modification regulates the transcriptional activity of XBP1. The Biochemical journal. 2010; 429: 95-102.

22. Jiang Z, Fan Q, Zhang Z, Zou Y, Cai R, Wang Q, et al. SENP1 deficiency promotes ER stress-induced apoptosis by increasing XBP1 SUMOylation. Cell cycle. 2012; 11: 1118-22.

23. Wang FF, Liu MZ, Sui Y, Cao Q, Yan B, Jin ML, et al. Deficiency of SUMO-specific protease 1 induces arsenic trioxide-mediated apoptosis by regulating XBP1 activity in human acute promyelocytic leukemia. Oncology letters. 2016; 12: 3755-62.

24. Wang H, Vinnikov I, Shahzad K, Bock F, Ranjan S, Wolter J, et al. The lectin-like domain of thrombomodulin ameliorates diabetic glomerulopathy via complement inhibition. Thrombosis and haemostasis. 2012; 108: 1141-53.

25. Madhusudhan T, Wang H, Straub BK, Grone E, Zhou Q, Shahzad K, et al. Cytoprotective signaling by activated protein $\mathrm{C}$ requires protease-activated receptor-3 in podocytes. Blood. 2012; 119: 874-83.

26. Dong W, Wang H, Shahzad K, Bock F, Al-Dabet MM, Ranjan S, et al. Activated Protein C Ameliorates Renal Ischemia-Reperfusion Injury by Restricting Y-Box Binding Protein-1 Ubiquitination. Journal of the American Society of Nephrology: JASN. 2015; 26: 2789-99.

27. Wang B, Nie J, Wu L, Hu Y, Wen Z, Dong L, et al. AMPKalpha2 Protects Against the Development of Heart Failure by Enhancing Mitophagy via PINK1 Phosphorylation. Circulation research. 2018; 122: 712-29.

28. Isermann B, Vinnikov IA, Madhusudhan T, Herzog S, Kashif M, Blautzik J, et al. Activated protein $C$ protects against diabetic nephropathy by inhibiting endothelial and podocyte apoptosis. Nature medicine. 2007; 13: 1349-58.

29. Madhusudhan T, Wang H, Ghosh S, Dong W, Kumar V, Al-Dabet MM, et al. Signal integration at the PI3K-p85-XBP1 hub endows coagulation protease activated protein C with insulin-like function. Blood. 2017; 130: 1445-55.

30. Hay RT. SUMO: a history of modification. Molecular cell. 2005; 18: 1-12.

31. Rytinki MM, Kaikkonen S, Pehkonen P, Jaaskelainen T, Palvimo JJ. PIAS proteins: pleiotropic interactors associated with SUMO. Cellular and molecular life sciences : CMLS. 2009; 66: 3029-41.

32. Wen I, Huang C. Coxsackieviruses B3 infection of myocardial microvascular endothelial cells activates fractalkine via the ERK1/2 signaling pathway. Molecular medicine reports. 2017; 16: 7548-52.

33. Ti Y, Xie GL, Wang ZH, Bi XL, Ding WY, Wang J, et al. TRB3 gene silencing alleviates diabetic cardiomyopathy in a type 2 diabetic rat model. Diabetes. 2011; 60: 2963-74.

34. Chen JH, Zhang P, Chen WD, Li DD, Wu XQ, Deng R, et al. ATM-mediated PTEN phosphorylation promotes PTEN nuclear translocation and autophagy in response to DNA-damaging agents in cancer cells. Autophagy. 2015; 11: 239-52. 
35. Thompson LM, Aiken CT, Kaltenbach LS, Agrawal N, Illes K, Khoshnan A, et al. IKK phosphorylates Huntingtin and targets it for degradation by the proteasome and lysosome. The Journal of cell biology. 2009; 187: 1083-99.

36. Rose BA, Force T, Wang Y. Mitogen-activated protein kinase signaling in the heart: angels versus demons in a heart-breaking tale. Physiological reviews. 2010; 90: 1507-46.

37. Lips DJ, Bueno OF, Wilkins BJ, Purcell NH, Kaiser RA, Lorenz JN, et al. MEK1-ERK2 signaling pathway protects myocardium from ischemic injury in vivo. Circulation. 2004; 109: 1938-41.

38. Wang A, Zhang H, Liang Z, Xu K, Qiu W, Tian Y, et al. U0126 attenuates ischemia/reperfusion-induced apoptosis and autophagy in myocardium through MEK/ERK/EGR-1 pathway. European journal of pharmacology. 2016; 788: 280-5.

39. Jo SK, Cho WY, Sung SA, Kim HK, Won NH. MEK inhibitor, U0126, attenuates cisplatin-induced renal injury by decreasing inflammation and apoptosis. Kidney international. 2005; 67: 458-66.

40. Larsson SC, Wallin A, Hakansson N, Stackelberg O, Back M, Wolk A. Type 1 and type 2 diabetes mellitus and incidence of seven cardiovascular diseases. International journal of cardiology. 2018; 262: 66-70.

41. Kanamori H, Takemura G, Goto K, Tsujimoto A, Mikami A, Ogino A, et al. Autophagic adaptations in diabetic cardiomyopathy differ between type 1 and type 2 diabetes. Autophagy. 2015; 11: 1146-60.

42. Marcovecchio ML, Chiesa ST, Bond S, Daneman D, Dawson S, Donaghue KC, et al. ACE Inhibitors and Statins in Adolescents with Type 1 Diabetes. The New England journal of medicine. 2017; 377: 1733-45.

43. Wang ZV, Deng Y, Gao N, Pedrozo Z, Li DL, Morales CR, et al. Spliced X-box binding protein 1 couples the unfolded protein response to hexosamine biosynthetic pathway. Cell. 2014; 156: 1179-92.

44. Duan Q, Ni L, Wang P, Chen C, Yang L, Ma B, et al. Deregulation of XBP1 expression contributes to myocardial vascular endothelial growth factor-A expression and angiogenesis during cardiac hypertrophy in vivo. Aging cell. 2016; 15: 625-33.

45. Bugger H, Abel ED. Rodent models of diabetic cardiomyopathy. Disease models \& mechanisms. 2009; 2: 454-66.

46. Fuentes-Antras J, Picatoste B, Gomez-Hernandez A, Egido J, Tunon J, Lorenzo O. Updating experimental models of diabetic cardiomyopathy. Journal of diabetes research. 2015; 2015: 656795.

47. Riehle C, Bauersachs J. Of mice and men: models and mechanisms of diabetic cardiomyopathy. Basic research in cardiology. 2018; 114: 2.

48. Miragoli M, Ceriotti P, Iafisco M, Vacchiano M, Salvarani N, Alogna A, et al. Inhalation of peptide-loaded nanoparticles improves heart failure. Science translational medicine. 2018; 10

49. Zuo G, Ren X, Qian X, Ye P, Luo J, Gao X, et al. Inhibition of JNK and p38 MAPK-mediated inflammation and apoptosis by ivabradine improves cardiac function in streptozotocin-induced diabetic cardiomyopathy. Journal of cellular physiology. 2019; 234: 1925-36.

50. Gu J, Wang S, Guo H, Tan Y, Liang Y, Feng A, et al. Inhibition of p53 prevents diabetic cardiomyopathy by preventing early-stage apoptosis and cell senescence, reduced glycolysis, and impaired angiogenesis. Cell death \& disease. 2018; 9: 82

51. Palmieri V, Tracy RP, Roman MJ, Liu JE, Best LG, Bella JN, et al. Relation of left ventricular hypertrophy to inflammation and albuminuria in adults with type 2 diabetes: the strong heart study. Diabetes care. 2003; 26: 2764-9.

52. Swoboda PP, McDiarmid AK, Erhayiem B, Ripley DP, Dobson LE, Garg P, et al. Diabetes Mellitus, Microalbuminuria, and Subclinical Cardiac Disease: Identification and Monitoring of Individuals at Risk of Heart Failure. Journal of the American Heart Association. 2017; 6.

53. Chandramouli C, Reichelt ME, Curl CL, Varma U, Bienvenu LA, Koutsifeli P, et al. Diastolic dysfunction is more apparent in STZ-induced diabetic female mice, despite less pronounced hyperglycemia. Scientific reports. 2018; 8: 2346.

54. Wang XM, Wang YC, Liu XJ, Wang Q, Zhang CM, Zhang LP, et al. BRD7 mediates hyperglycaemia-induced myocardial apoptosis via endoplasmic reticulum stress signalling pathway. Journal of cellular and molecular medicine. 2017; 21: 1094-105.

55. Shao D, Liu J, Ni J, Wang Z, Shen Y, Zhou L, et al. Suppression of XBP1S mediates high glucose-induced oxidative stress and extracellular matrix synthesis in renal mesangial cell and kidney of diabetic rats. PloS one. 2013; 8: e56124.

56. Shao $\mathrm{D}, \mathrm{Ni}$, Shen $\mathrm{Y}$, Liu J, Zhou $\mathrm{L}$, Xue $\mathrm{H}$, et al CHOP mediates XBP1S-induced renal mesangial cell necrosis following high glucose treatment. European journal of pharmacology. 2015; 758: 89-96.

57. Park SW, Zhou Y, Lee J, Lu A, Sun C, Chung J, et al. The regulatory subunits of PI3K, p85alpha and p85beta, interact with XBP-1 and increase its nuclear translocation. Nature medicine. 2010; 16: 429-37.

58. Yang L, Calay ES, Fan I, Arduini A, Kunz RC, Gygi SP, et al. METABOLISM. S-Nitrosylation links obesity-associated inflammation to endoplasmic reticulum dysfunction. Science. 2015; 349: 500-6.

59. Yao $Q$, Li H, Liu BQ, Huang XY, Guo L. SUMOylation-regulated protein phosphorylation, evidence from quantitative phosphoproteomics analyses. The Journal of biological chemistry. 2011; 286: 27342-9.

60. Zhang J, Zhao B, Zhu X, Li J, Wu F, Li S, et al. Phosphorylation and SUMOylation of CRMP2 regulate the formation and maturation of dendritic spines. Brain research bulletin. 2018; 139: 21-30.
61. Nie Q, Gong XD, Liu M, Li DW. Effects of Crosstalks Between Sumoylation and Phosphorylation in Normal Cellular Physiology and Human Diseases. Current molecular medicine. 2017; 16: 906-13.

62. Hietakangas V, Anckar J, Blomster HA, Fujimoto M, Palvimo JJ, Nakai A, et al. PDSM, a motif for phosphorylation-dependent SUMO modification. Proceedings of the National Academy of Sciences of the United States of America. 2006; 103: 45-50.

63. Gao J, Shao K, Chen X, Li Z, Liu Z, Yu Z, et al. The involvement of post-translational modifications in cardiovascular pathologies: Focus on SUMOylation, neddylation, succinylation, and prenylation. Journal of molecular and cellular cardiology. 2020; 138: 49-58.

64. Celen AB, Sahin U. Sumoylation on its 25th anniversary: mechanisms, pathology, and emerging concepts. The FEBS journal. 2020.

65. Liu J, Ibi D, Taniguchi K, Lee J, Herrema H, Akosman B, et al. Inflammation Improves Glucose Homeostasis through IKKbeta-XBP1s Interaction. Cell. 2016; 167: 1052-66 e18.

66. Jiao FJ, Wang QZ, Zhang P, Yan JG, Zhang Z, He F, et al. CDK5-mediated phosphorylation of $\mathrm{XBP} 1 \mathrm{~s}$ contributes to its nuclear translocation and activation in $\mathrm{MPP}(+)$-induced Parkinson's disease model. Scientific reports. 2017; 7: 5622.

67. Wang $\mathrm{Y}$, Zhang $\mathrm{Y}$, Yi $\mathrm{P}$, Dong $\mathrm{W}$, Nalin AP, Zhang $\mathrm{J}$, et al. The IL-15-AKT-XBP1s signaling pathway contributes to effector functions and survival in human NK cells. Nature immunology. 2019; 20: 10-7.

68. Bailey KA, Moreno E, Haj FG, Simon SI, Passerini AG. Mechanoregulation of p38 activity enhances endoplasmic reticulum stress-mediated inflammation by arterial endothelium. FASEB journal : official publication of the Federation of American Societies for Experimental Biology. 2019; 33: 12888-99.

69. Wauson EM, Guerra ML, Barylko B, Albanesi JP, Cobb MH. Off-target effects of MEK inhibitors. Biochemistry. 2013; 52: 5164-6. 